Miriam Morek

\title{
Familien- und Peer-Interaktionen als Erwerbsressource für Diskurskompetenzen
}

\author{
Empirische Befunde zu Variabilität und Erwerbspotenzial \\ außerschulischer Diskurspraktiken von Präadoleszenten
}

\begin{abstract}
Ziel dieses Beitrags ist es, empirisch vorfindbare Unterschiede in den außerschulischen Diskurspraktiken von Präadoleszenten zu beschreiben und sie auf ihr Potenzial als externe Ressource des kindlichen Diskurserwerbs zu befragen. $\mathrm{Zu}$ diesem Zweck werden Ergebnisse einer qualitativen, gesprächsanalytischen Studie präsentiert, in der Familien- und Peer-Interaktionen derselben 10-Jährigen aufgezeichnet und mit Blick auf Auftreten und interaktive Ausgestaltung globaler Diskurspraktiken untersucht wurden. In einem ersten Schritt werden in einer überblicksartigen ,Draufsicht‘ zunächst Auftreten und Verteilungen von Diskurspraktiken in den je 12 Familien und Cliquen berichtet (Fokus: Erzählen, Argumentieren, Erklären); hier zeigen sich - v. a. milieuabhängig - teils drastische Unterschiede. In einer kontrastiv angelegten Fallstudie zu zwei Kindern werden die Befunde sodann vertieft: Mit Blick auf das Argumentieren in Familien- und das Erklären in Cliquen-Interaktionen wird nachgezeichnet, auf welche Weise die Interaktanten diskursive Erwerbskontexte, die sich in ihrer Reichhaltigkeit und Erwerbsförderlichkeit deutlich unterscheiden, mikrogenetisch hervorbringen. Aus den beobachteten Gesprächsprozessen wird die These abgeleitet, dass Kinder unter Nutzung ihrer internen Ressourcen durch ihre Gesprächsaktivitäten selbst mitbeeinflussen, welche externen Ressourcen ihnen im Bereich interaktiver Unterstützung durch Mitinteraktanten zur Verfügung stehen.
\end{abstract}

Keywords: Familieninteraktion, Peer-Interaktion, Sprachsozialisation, Heterogenität, Ressourcen des Diskurserwerbs, Argumentieren, Erklären

Miriam Morek, Universität Duisburg-Essen, Universitätsstr. 12, 45141 Essen, miriam.morek@uni-due.de

Ә Open Access. (C 2021 Miriam Morek, publiziert von De Gruyter. (c) BY-NC-ND Dieses Werk ist lizensiert unter einer Creative Commons Namensnennung - Nicht-kommerziell - Keine Bearbeitung 4.0 International Lizenz. 


\section{Einleitung: Spracherwerb und Sprachsozialisation}

Dass soziale Interaktionen eine wesentliche Rolle bei der kindlichen Aneignung sprachlicher Fähigkeiten spielen (z. B. Bruner 1983; Slobin, Tripp \& Silverman 1996; Snow 1977), kann inzwischen als Konsens der Spracherwerbsforschung gelten (z. B. Arnon et al. 2014; Behrens 2014; Clark 2017; Snow 2014; Stoll 2016). Dies gilt umso mehr für den Bereich diskursiver Kompetenzen: Die Fähigkeit, komplexe sprachliche Praktiken wie Erzählen, Argumentieren oder Erklären in Gesprächen vollziehen zu können, wird empirisch nachweisbar wesentlich über Muster der Erwachsenen-Kind-Interaktion erworben (Hausendorf \& Quasthoff 1996; Kern \& Quasthoff 2007; Heller \& Krah 2015; Quasthoff \& Kluger in diesem Band). Die Spracherwerbsforschung hat allerdings - in ihrem Bemühen um verallgemeinerbare Aussagen über Erwerbsverläufe - die Variabilität sozialer Bedingungen des Erwerbs weitgehend unberücksichtigt gelassen (Kurumada \& Arnon 2014: 3; Nardy, Chevrot \& Barbu 2013: 257; Nelson 2014: 101; vgl. aber schon früh: Hart \& Risley 1995). Nicht zuletzt die Einsichten der Bildungsforschung zur zentralen Rolle sprachlicher Fähigkeiten für den Bildungserfolg rücken jedoch die Frage nach der Unterschiedlichkeit alltäglicher Sprachund Interaktionserfahrungen von Kindern und Jugendlichen in den Fokus der Aufmerksamkeit (Snow 2014: 117; vgl. Quasthoff \& Kluger in diesem Band). Einen wichtigen Bezugspunkt bildet dafür die Sprachsozialisationsforschung (Duranti, Ochs \& Schieffelin 2012; Ochs \& Schieffelin 2012; Paugh 2016). In ihrer anthropologischen oder soziolinguistischen Ausrichtung nimmt sie, anders als die Spracherwerbsforschung, die jeweiligen soziokulturellen Kontexte sprachlicher Aneignungsprozesse zentral in den Blick (Ochs \& Schieffelin 2012: 1-2; für einen Überblick vgl. Duranti, Ochs \& Schieffelin 2012). Sprachsozialisation wird dabei in einem zweifachen Sinne verstanden als „socialization through the use of language and socialization to use language“ (Schieffelin \& Ochs 1986: 163). Wie unterschiedlich sich letzteres, also die Sozialisierung in bestimmte sprachlich-kommunikative Praktiken gestalten kann, zeigen v.a. ethnographische (Heath 1983; Isler 2014; Lareau 2003) und gesprächsanalytische Arbeiten (Heller 2012; Kern \& Quasthoff 2007; Morek 2012). Sie fokussieren bislang v.a. sprachliche Alltagsroutinen in Familien.

Die Frage, ob und inwiefern darüber hinaus auch Peer-Interaktionen mit Gleichaltrigen ein Kontext für den kindlichen Ausbau sprachlicher und diskursiver Fähigkeiten sein können, wird allerdings in jüngerer Zeit verstärkt in den Blick genommen (z. B. Arendt 2015; Blum-Kulka \& Snow 2004; Cekaite et al. 
2014; Morek 2014, 2015; Stude 2013). Insgesamt also werden zusehends unterschiedliche Kontexte des Erwerbs sprachlich-diskursiver Fähigkeiten betrachtet.

Allerdings ist das gegenwärtige Wissen über interindividuelle Varianzen solcher externer Erwerbsressourcen (vgl. zu diesem und anderen grundlegenden Konzepten das Glossar in diesem Band), wie sie mit Familien- und Peer-Interaktionen potenziell zur Verfügung stehen, begrenzt. Insbesondere ist bislang kaum bekannt, ob bzw. wie Peer-Interaktionen verschiedener Kinder differieren (vgl. aber Morek 2014). Auch ist die Frage noch unbeantwortet, wie sich familiale und amikale Interaktionserfahrungen einzelner Kinder zu einer je individuellen Konstellation außerschulisch verfügbarer externer Ressourcen des Diskurserwerbs zusammenfügen.

Der vorliegende Beitrag unternimmt einen Versuch, diese Fragen im Sinne eines (kindbezogenen) Kontextvergleiches anzugehen, der Familien- und Peer-Interaktionen unter Gesichtspunkten diskursiver Praktiken beleuchtet; im Fokus stehen dabei die Praktiken Argumentieren und Erklären. Präsentiert werden ausgewählte Befunde aus dem Projekt DisKo (,Diskursive Praktiken von Kindern in außerschulischen und schulischen Kontexten“), das an der TU Dortmund unter der Leitung von Uta Quasthoff durchgeführt wurde (vgl. Quasthoff in diesem Band). Diese kontextvergleichend angelegte Studie untersuchte die Diskurspraktiken derselben Fünftklässler in Familien-, Peer- und Unterrichtsinteraktionen. Im Fokus stand dabei die Frage nach der kindlichen Fähigkeit, an kommunikativ und sozial sehr unterschiedlich situierten Diskurspraktiken sprachlich angemessen partizipieren zu können (vgl. dazu genauer: Quasthoff \& Morek 2015). Zu diesem Zweck wurden natürliche Interaktionen von 12 Präadoleszenten unterschiedlicher sozialer Hintergründe in den o. g. drei Settings aufgezeichnet (Gesamtumfang: ca. 90 Stunden; für eine genauere Beschreibung des empirischen Vorgehens in DisKo s. Quasthoff in diesem Band). Der vorliegende Beitrag betrachtet die Teilkorpora ,Familiengespräche‘ und ,Cliquengespräche' und fokussiert die Frage, wie sich die dortigen alltäglichen Interaktionserfahrungen der untersuchten Kinder unterscheiden, und zwar unter dem Gesichtspunkt ihrer Erwerbsfunktionalität für schulrelevante Diskurskompetenzen. Er ergänzt damit die Beiträge von Quasthoff et al. (in diesem Band), Quasthoff \& Kluger (in diesem Band) sowie Domenech \& Krah (in diesem Band), die sich - am Beispiel des Argumentationserwerbs - speziell auf familiale Ressourcen konzentrieren.

Aufgebaut ist der Beitrag wie folgt: Abschnitt 2 liefert die theoretische Grundlegung: das hier zugrunde gelegte Modell diskursiver Kompetenzen (vgl. auch Quasthoff, Heller \& Morek in diesem Band) und die Unterscheidung externer und interner Ressourcen des Diskurserwerbs. Anschließend werden bisherige Befunde zur Unterschiedlichkeit außerschulischer externer Ressourcen skizziert, aus denen die Leitfragen für diesen Beitrag abgeleitet werden. Abschnitt 3 stellt 
Anlage, Datenerhebung und analytischen Zugang des DisKo-Projekts vor. In Abschnitt 4 wird zunächst überblicksartig über die Diskurspraktiken in den Familienund Peer-Interaktionen der untersuchten 10-12-Jährigen berichtet. In Abschnitt 5 werden die Ergebnisse anhand zweier ausgewählter Fokuskinder und ihren Familien- und Cliquen-Interaktionen konkretisiert und exemplarisch vorgeführt. Das Fazit (Abschnitt 6) plädiert abschließend ausgehend von den analytischen Befunden für eine Dynamisierung des Konzepts ,Erwerbskontext', insbesondere mit Blick auf spätere Phasen des Diskurserwerbs.

\section{Altagsinteraktionen als Diskurserwerbsressource}

\subsection{Interne und externe Ressourcen des Erwerbs von Diskurskompetenz}

Unter Diskurskompetenz wird in diesem Beitrag die Fähigkeit verstanden, an komplexen mündlichen Kommunikationspraktiken partizipieren zu können, deren Geordnetheit das kontingente Aufeinanderfolgen zweier Äußerungen (etwa: Frage Antwort) übersteigt, die also die interaktive Herstellung ,größerer sequenzieller Zusammenhänge erfordern (vgl. das Glossar in diesem Band). Prototypisch zählt dazu etwa die Fähigkeit, in Gesprächen erzählen, argumentieren oder etwas erklären zu können. Bei all diesen Aktivitäten geht es um den produktiven und rezeptiven Umgang mit sog. „globalen Zugzwängen“ (Hausendorf \& Quasthoff 1996), deren Bearbeitung jeweils den sequenziellen, interaktiven Vollzug der entsprechenden Gattung steuert (Quasthoff, Heller \& Morek 2017).

Modelliert werden kann Diskurskompetenz entlang dreier Aufgabenbereiche bzw. Teilfähigkeiten (vgl. Quasthoff, Heller \& Morek in diesem Band): (1) Die Kontextualisierung betrifft die Organisation der jeweiligen Diskursaktivität auf der Ebene der gemeinsamen, globalen Interaktionsaufgaben (Ohlhus \& Stude 2009: 474). Hier geht es darum, eine Diskursaktivität passend in ein laufendes Gespräch einzubetten (z. B. mit Blick auf Beteiligungsrollen, interaktive Teilaufgaben, kommunikativen Zweck, Thema). Differenziert werden kann dabei eine sequenzielle und eine soziale Seite des Kontextualisierens (Morek \& Quasthoff 2018): Die sequenzielle richtet sich auf das Management struktureller Anschlussstellen in einer Interaktion, die soziale hingegen bezieht sich auf die Aufgabe, eine Diskursaktivität gemäß den Anforderungen und Normen eines bestimmten sozialen Kontexts zuzuschneiden. (2) Bei der Vertextung geht es um den inneren Aufbau einer diskursiven Einheit (z. B. einer Erzählung, einer Argumentation). Sie folgt i.d.R. gattungsspezi- 
fischen Anforderungen und verlangt die Auswahl und Anordnung inhaltlicher Elemente (z. B. Setting und Höhepunkt beim Erzählen) in Relation zueinander (vgl. dazu Heller in diesem Band). (3) Das dritte Aufgabenfeld schließlich, die Markierung, bezieht sich auf die Ebene konkreter sprachlicher (und nicht-sprachlicher) Formen, mit denen die jeweilige Gattung und ihr Aufbau indiziert werden.

Diskurskompetenz ist also eine ausgesprochen komplexe Fähigkeit. Ihre Aneignung zieht sich bis mindestens in die Adoleszenz hinein (vgl. Nippold 2006; Quasthoff \& Kluger in diesem Band). Ressourcen dieses Erwerbs können in interne und externe unterschieden werden (Kern 2011; Quasthoff 2011; Quasthoff et al. 2019). Unter ersteren werden Kompetenzen verstanden, die dem Kind zu einem Zeitpunkt im Erwerbsprozess als kontextfreie Ressourcen zur Verfügung stehen (Quasthoff et al. 2019). Dazu zählen neben den o.g. diskursiven Teilfähigkeiten einschließlich des relevanten Gattungswissens auch allgemeine kognitive Fähigkeiten sowie sprachliches Strukturwissen.

Als externe Ressourcen lassen sich dagegen verschiedene Ausprägungen kontextueller, also ,von außen“ gelieferter Unterstützung fassen (vgl. Quasthoff et al. 2019). Darunter fallen zuallererst Interaktionserfahrungen eines Kindes und zwar hier v. a. solche mit kompetenteren Erwachsenen -, die beim Vollzug entsprechender Diskursaktivitäten implizite Hilfen bieten und als Discourse Acquisition Support System (Hausendorf \& Quasthoff 1996) fungieren können. Es lassen sich unterschiedlich erwerbssupportive Interaktionsmuster unterscheiden, die sich in den eltern- und kindseitigen Anteilen sowie in der Art und Weise dokumentieren, wie die kommunikativen Teilaufgaben (Jobs) einzelner Diskursaktivitäten bearbeitet werden (vgl. dazu Quasthoff \& Kluger in diesem Band). In dem Maße, wie Diskurserwerb sich über die Beteiligung des Kindes an entsprechenden Praktiken vollzieht, geht es mit Blick auf habitualisierte kommunikative Erfahrungen grundlegend auch um die Frage, welche diskursiven Praktiken einem Kind überhaupt regelhaft - ob produktiv oder rezeptiv - zur Verfügung stehen. Diesen Aspekt hat Heller (2012: 106ff.) über die Bestimmung sog. Gattungsrepertoires (Günthner 2009) erfasst. Neben der interaktiven Involvierung in den Vollzug entsprechender Gattungen kommt so auch die Rezeption von Modellen als weiterer potenzieller Mechanismus externer Erwerbsunterstützung ins Spiel (Ohlhus \& Stude 2009; Quasthoff 2011; vgl. auch Kleinschmidt-Schinke 2018). ${ }^{1}$

Im Fokus des vorliegenden Beitrags stehen die externen Erwerbsressourcen in Form habitualisierter interaktiver Erfahrungen in Familien und Cliquen. Allerdings

1 Daneben zählt auch Instruktion, d.h. die explizite Thematisierung von Diskursaktivitäten als Lerngegenstand zu den externen Ressourcen (Ohlhus \& Stude 2009). Sie spielt allerdings in informell-privaten Interaktionen eine untergeordnete Rolle. 
ließ sich in einer Studie zur Diskurs- und Textproduktionsentwicklung von Grundschulkindern empirisch nachzeichnen, dass „nicht allein die Unterschiedlichkeit der jeweiligen Erwerbskontexte ausschlaggebend für individuell verlaufende Erwerbsprozesse“ ist (Kern 2011: 249). Vielmehr zeigte sich, dass die Ontogenese von Diskursfähigkeiten als Integration interner und externer Ressourcen modelliert werden muss (Quasthoff 2011; Quasthoff et al. 2019): Kinder bringen Vorwissen, schon vorhandene Diskursfähigkeiten und weitere kognitive Ressourcen ein, um auf dieser Basis die ihnen jeweils (z. B. als Modell oder Instruktion) zur Verfügung stehenden externen Ressourcen nutzen $\mathrm{zu}$ können. Folgt man dieser Einsicht, dann ist es plausibel, davon auszugehen, dass ein solches Zusammenspiel von internen und externen Ressourcen auch auf mikrogenetischer (Quasthoff 2012) Basis relevant wird. Denkbar ist, dass Kinder auf Basis ihrer internen Ressourcen in der interaktiv konstituierten Vollzugswirklichkeit konkreter Gesprächskontexte mitbestimmen, wie die ihnen zur Verfügung stehenden externen Ressourcen (z. B. die eingebrachte erwachsenseitige Unterstützung) aussehen. Diese Überlegungen gründen sich auf ein sozialkonstruktivistisches Verständnis von ,Kontext‘ (vgl. 2.2).

\subsection{Zur Heterogenität außerschulischer Kontexte des Diskurserwerbs}

Legt man ein sozialkonstruktivistisches, dynamisch-reflexives Verständnis von ,Kontext‘ zugrunde (Auer 1986; Garfinkel 1967; Gumperz 1982), sind auch Erwerbskontexte nicht einfach ,da“ - wie etwa die Rede vom „Sprachinput“ im Rahmen der sprachstrukturell und der quantitativ orientierten Spracherwerbsforschung nahelegt (z. B. Stoll 2016; vgl. Kleinschmidt-Schinke 2018). Vielmehr werden sie interaktiv hergestellt, nämlich durch die je spezifische Art und Weise, wie Gesprächsbeteiligte - z. B. Eltern und Kindern oder Kinder und ihre Peers sprachlich miteinander agieren. Ein so verstandener Kontext kann von unterschiedlicher Reichweite sein und sich von lokal-sequenziellen Kontexten aus Vorgänger- und Nachfolgeäußerung über globale Diskursaktivitäten bis hin zu ganzen Interaktionssituationen bzw. wiederkehrenden „sozialen Veranstaltungen“ (Luckmann 1989) in einer bestimmten Interaktionsgemeinschaft erstrecken. Als soziale Veranstaltungen (z. B. gemeinsame Mahlzeiten, Fernsehabend) lassen sich mit Luckmann (1989) regelmäßig wiederkehrende Zusammenkünfte von sozial oder institutionell miteinander verbundenen Personen verstehen, bei denen wiederkehrende, dem jeweiligen Zusammenschluss eigene kommunikative ,Probleme' bearbeitet werden. Nach wissenssoziologischem bzw. praxeologischem Verständnis werden dabei jeweils bestimmte Sprach- und Diskurspraktiken rekurrent relevant gesetzt (Günthner \& Christmann 1996) und auch bestimmte thematische 
Präferenzen kultiviert (Heller 2012). Auf diese Weise ergeben sich gruppen- oder milieuspezifische Themen- und Gattungsrepertoires (Günthner 2009), die wiederum unterschiedlich weite kommunikative Erfahrungsräume für den Erwerb sprachlicher und diskursiver Fähigkeiten bereitstellen.

So wurde mit Blick auf unterrichtsrelevante Sprach- und Diskurserfahrungen von Kindern gezeigt, dass in Familien z. B. das unterrichtsrelevante Erklären (Morek 2012) und Argumentieren (Heller 2012; Lareau 2003) gerade nicht Teil des Gattungsrepertoires jeder Familie sind. Auf mikroanalytischer Ebene konnte außerdem detailliert rekonstruiert werden, dass und wie die jeweils v. a. durch bestimmte elterliche Gesprächsaktivitäten geprägten Interaktionsmuster beim Vollzug entsprechender Diskurspraktiken variieren (vgl. Heller 2012; Heller \& Krah 2015; Kern \& Quasthoff 2007; Morek 2012; Quasthoff \& Krah 2012). Kurz gefasst erweist sich grundsätzlich ein familiales Interaktionsmuster als besonders erwerbsförderlich, das dem Kind viel Gesprächsraum für eigene diskursive Beiträge zuweist (z. B. Warum-Frage zur Etablierung argumentativer Anforderungen) und es bei deren Vollzug interaktiv unterstützt (z. B. durch klärende Nachfragen, Hörersignale, Einwände). Ungleich weniger förderlich als dieses Fordern und Unterstützen wirken sich dagegen Interaktionsmuster aus, die keine besonderen diskursiven Anforderungen an Kinder etablieren („Raumlassen und Akzeptieren“ bzw. „Dulden und Tilgen“; Morek 2012; Heller 2012) oder sie primär zu Adressaten elternseitiger Argumentations- und Erklärbemühungen machen, statt sie produktiv zu involvieren („Übergehen und Selberlösen“, Quasthoff \& Krah 2012). Für eine ausführliche Darstellung solcher Varianzen sei an dieser Stelle auf den Beitrag von Quasthoff \& Kluger (in diesem Band) verwiesen.

Vergleichbare Befunde $\mathrm{zu}$ Gattungsrepertoires und Interaktionsmustern verschiedener Peergroups fehlen bislang, was u. a. auch mit den besonderen Herausforderungen bei der Erhebung entsprechender Vergleichsdaten verbunden sein dürfte (Quasthoff \& Morek 2015; vgl. Quasthoff in diesem Band). Nur vereinzelt liegen Hinweise auf die Existenz sprachlich-kommunikativer Varianzen in der Face-to-Face-Kommunikation verschiedener Cliquen vor (Schlobinski, Kohl \& Ludewigt 1993 zu Sprechstilen; Walther 2014 zu genderspezifischen Läster- und Frotzelaktivitäten). Für die Frage nach unterrichtsrelevanten Diskurserfahrungen ist die Studie von Eder (1990) einschlägig; sie berichtet für amerikanische Teenager schichtspezifische Präferenzen einer eher ernsthaften Konfliktaustragung bei „girls from middleclass backgrounds“ bzw. einer spielerisch-aggressiven Variante i.S. ritueller Konflikte (,girls from working- or lower-class backgrounds“) (Eder 1990: 74). Systematisch mit Blick auf Potenziale von Diskurserwerb reflektiert wurden solche Einblicke in die Unterschiedlichkeit sprachlicher Peer-Praktiken bislang jedoch i.d. R. nicht (vgl. aber Morek 2014, 2015). 


\subsection{Fragestellung des Beitrags}

Insgesamt also lässt sich festhalten, a) dass habitualisierte Interaktionserfahrungen von Kindern in ihren alläglichen Sozialisationskontexten eine entscheidende externe Ressource des Diskurserwerbs darstellen, b) dass solche Erfahrungen in verschiedener Hinsicht variieren. Offen ist indes die Frage, wie solche externen Ressourcen gerade im Hinblick auf den Erwerb bildungssprachlicher Diskurskompetenzen für einzelne Kinder konkret konstelliert sind und vor allem, wie Peer- im Vergleich zu Familieninteraktionen unter Ressourcengesichtspunkten einzuordnen sind. Hierzu bedarf es eines systematisch kontextvergleichenden Zugriffs. In diesem Sinne fragt der vorliegende Beitrag:

1. Wie unterscheiden sich habitualisierte Interaktionserfahrungen unterschiedlicher Präadoleszenter in ihren Familien und Cliquen, und zwar mit Blick auf

a. praktizierte Genres,

b. interaktive erwerbssupportive Unterstützung von Mitinteraktanten

c. sowie Repertoires an semantisch-pragmatischen Mitteln und deren Vertextung sowie sprachlicher (und nicht-sprachlicher) Formen (vgl. Abschnitt 3)?

2. Welche Bezüge bestehen zwischen externen und internen Ressourcen bzw. wie tragen Lernerinnen und Lerner ihrerseits zu ihren eigenen kontextuellen Erwerbsressourcen bei?

Beantwortet werden diese Fragen vor dem Hintergrund der Analyse des Korpus (vgl. Abschnitt 4) v. a. mit Hilfe eines exemplarischen Fallvergleichs (vgl. Abschnitt 5).

\section{Daten und analytischer Zugang}

Um außerschulische Sprach(sozialisations)erfahrungen unterschiedlicher Kinder in ihren Familien und Cliquen vergleichend untersuchen zu können, musste das Projekt DisKo zunächst die methodische Herausforderung lösen, Feldzugang zu diesen sehr privaten Domänen zu erlangen, und zwar in soziokulturell möglichst heterogenen Milieus. Zu diesem Zweck wurden über informelle Gespräche und Fragebögen in insgesamt 17 fünften Klassen unterschiedlicher Schulformen in zwei nordrheinwestfälischen Großstädten zunächst mögliche Cliquen rekrutiert (vgl. dazu genauer: Quasthoff \& Morek 2015), von denen 12 für die Teilnahme an der Studie ausgewählt wurden. Leitend dabei war, dass sowohl Gruppen sozial privilegierter als auch sozial benachteiligter Milieus vertreten sein sollten sowie ein möglichst 
großes Spektrum verschiedener kinder- bzw. jugendkultureller Orientierungen. ${ }^{2}$ Daher wurden sechs Cliquen aus Gymnasien (in Tab. 1: + privilegiert) ausgewählt sowie sechs aus Haupt- bzw. Gesamtschulen (in Tab. 1: - privilegiert). ${ }^{3}$ Die Cliquen waren entsprechend der Lebenswelt der Kinder bis auf eine Ausnahme gleichgeschlechtlich zusammengesetzt und umfassten zwischen drei und sieben Mitgliedern, die jeweils in dieselbe Schulklasse gingen (insgesamt: 53 Kinder zwischen 10 und 12 Jahren).

Um ungesteuerte, möglichst „natürliche“ (vgl. Quasthoff in diesem Band) Gesprächsdaten der Cliquen zu erhalten, dabei jedoch gemäß der Projektfragestellung auch Aufschluss über individuelle kommunikative Repertoires Einzelner zu erlangen, wurde in verschiedenen städtischen Jugendzentren jeweils ein zweigeteiltes Setting arrangiert: Im ersten, unter Forscherinnenanwesenheit stattfindenden Erhebungsteil wurde mit den Kindern jeweils eine Gruppendiskussion durchgeführt; außerdem füllten die Kinder Fragebögen aus, die u. a. Angaben zu Familiensprache(n), beruflichen Tätigkeiten der Eltern sowie zum Bücherbestand im Haushalt enthielten. Im anschließenden zweiten Erhebungsteil durften sich die Gruppen jeweils - ohne Aufsicht, aber bei laufenden Kameras - „zur Belohnung für ihre Teilnahme“ Pizza bei einem Lieferdienst bestellen. Für die Aufzeichnung ungesteuerter Ingroup-Kommunikation erwiesen sich die Zeit des Wartens auf die Lieferung sowie das gemeinsame Essen als zentral. Der Einschluss von Tischgesprächen mit ihrer naturgemäß meist räumlichen Bindung sorgte dabei in allen Gruppen für eine recht hohe Kommunikationsintensität und ermöglichte so den Vergleich des ,Sich-Unterhaltens“ in verschiedenen Cliquen. Insgesamt umfasst das Teilkorpus ,Cliquen' rund 22 Stunden an Videoaufzeichnungen (je 2 statische Kameras im Raum), wovon fast 15 Stunden Ingroup-Interaktion ohne Anwesenheit der Forschenden beinhalten.

In einem zweiten Schritt wurden aus den Cliquen 12 Kinder für die Aufzeichnung in ihren Familien ausgewählt, ${ }^{4}$ wobei wiederum die Berücksichtigung einer

2 Gemäß dem Projektfokus auf deutschsprachige Interaktionskontexte sollten außerdem solche Kinder und Cliquen ausgewählt werden, deren außerschulische Interaktionen sich i.d.R. auf Deutsch vollziehen. Diese Informationen - wie auch Auskünfte über gemeinsame Vorlieben und Hobbys der jeweiligen Clique - wurden per Fragebogen ermittelt.

3 Zum Zusammenhang von sozialer Herkunft und Schulform vgl. insbesondere Bonsen et al. (2008). 4 In Tab. 1 sind in der Spalte ,Fokuskinder' insgesamt 15 Namen aufgeführt. Dies ist dem Umstand geschuldet, dass im Verlauf des Projekts aufgrund von Schulwechsel oder Umzug einige ,Drop-Outs‘ zu verzeichnen waren, sodass drei Fokuskinder neu rekrutiert werden mussten, die auch für den dritten Erhebungsschritt, die Unterrichtsaufzeichnungen zur Verfügung standen. Die ursprünglich auch als Fokuskind ausgewählte ,Sabrina‘ wurde - zugunsten anderer Fokuskinder - im weiteren Projektverlauf nicht weiter verfolgt, da sich in ihren Unterrichtsinteraktionen insgesamt nur sehr wenige lehrerseitig geschaffene Diskursanlässe fanden. 


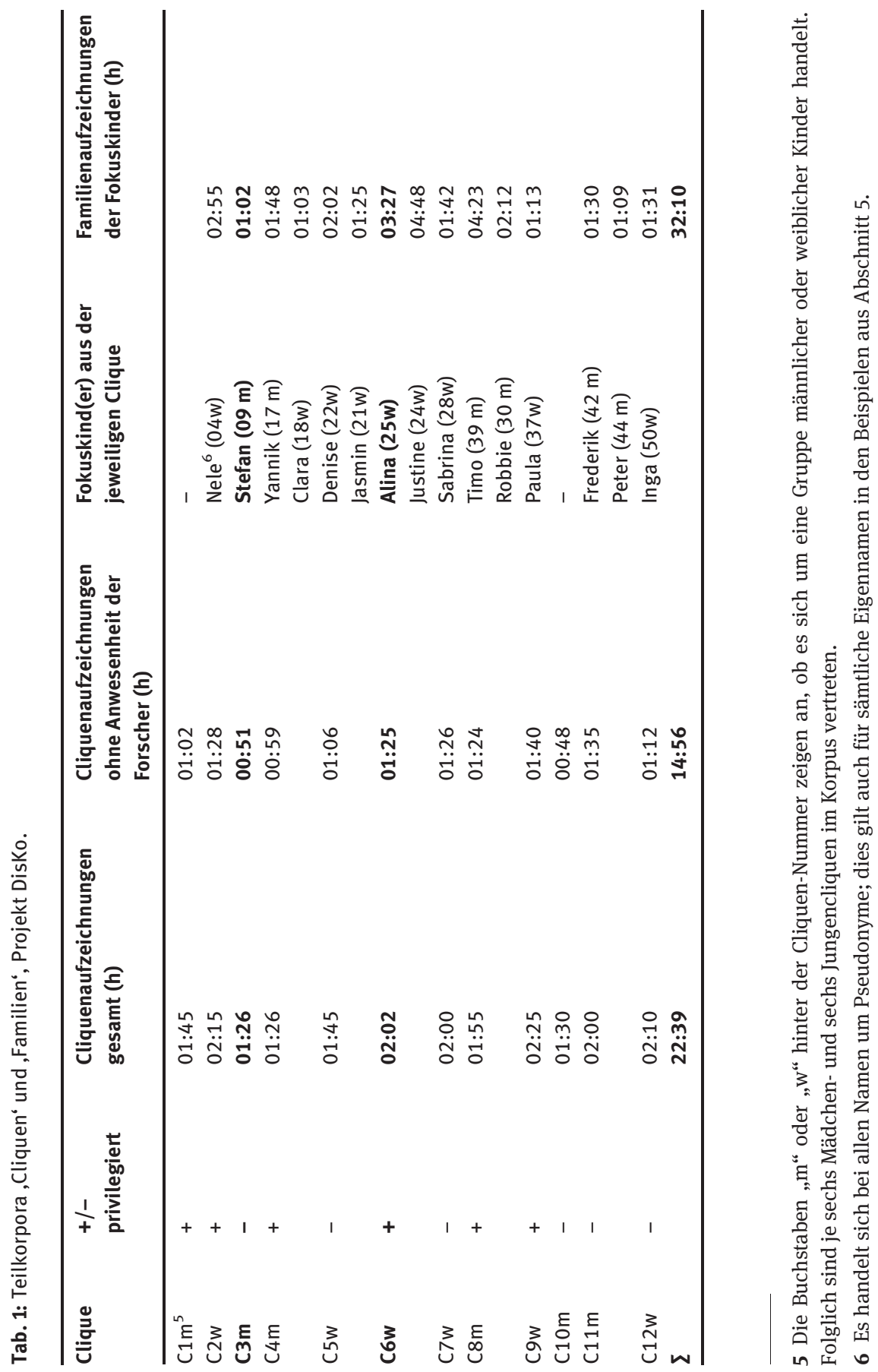


möglichst großen Bandbreite diskursiver und sozialer Aspekte leitend war. Mit Hilfe der Fragebögen und erster Videoanalysen wurden Kinder identifiziert, die sich v. a. im elterlichen sozialen Milieu, den individuell gezeigten Diskurskompetenzen sowie ihren Partizipationsmustern an den verschiedenen interaktiven Situationen des Cliquen-Settings unterschieden (vgl. dazu genauer: Quasthoff \& Morek 2015 sowie Quasthoff in diesem Band). Das Teilkorpus ,Familien“ besteht fast ausschließlich aus Mitschnitten von Tischgesprächen und umfasst rund 32 Stunden Audiodateien. ${ }^{7}$ Tabelle 1 gibt einen Überblick über die (außerschulische) Datenkonstellation aus DisKo. Die für den exemplarischen Fallvergleich ausgewählten Fokuskinder sind durch Fettdruck hervorgehoben.

Zur Vorbereitung der gesprächsanalytischen Untersuchung wurde zunächst das Cliquenkorpus gesichtet und hinsichtlich der in den ungesteuerten Erhebungsteilen vorkommenden Gesprächspraktiken kodiert. In diesem Schritt ging es darum, einen Überblick über die jeweiligen Gattungsrepertoires der verschiedenen Cliquen zu erlangen (vgl. Abschnitt 2.3). ${ }^{8}$ Weil die übergeordnete Projektfragestellung auf die Nutzbarkeit außerschulischer Diskurspraktiken im Hinblick auf schulische Erwartungen zielte, wurde im weiteren Projektverlauf ein Fokus auf explanative, argumentative sowie narrative Sequenzen gelegt, d. h. diejenigen „Gattungsfamilien“ (Luckmann 1989: 42), die immer wieder im Zusammenhang mit bildungs- bzw. schulsprachlichem Handeln angeführt werden (z. B. Schleppegrell 2004; Uccelli et al. 2014: 9). Als explanativ wurden dabei Sequenzen verstanden, in denen das Was, Wie oder Warum von Sachverhalten zum Zwecke des Transfers komplexen Wissens expliziert wird, als argumentativ hingegen solche, die die begründende, wechselseitige Aushandlung von Geltungsansprüchen bzw. divergierenden Positionen zum Gegenstand haben (Heller 2012; Morek, Heller \& Quasthoff 2017). Als narrativ schließlich wurden Sequenzen aufgefasst, in denen Ereignisabfolgen mit besonderer reportability (Labov \& Waletzky 1967) wiedergegeben werden (z. B. als Erzählung oder Bericht) (vgl. zusammenfassend auch Quasthoff, Heller \& Morek 2017).

Bezogen auf diese drei großen Gattungsfamilien wurden insgesamt etwa 380 Sequenzen (einschließlich der für diesen Beitrag nicht relevanten Unter-

7 Die Länge der pro Familie in Eigenregie erstellten Aufnahmen (Vorgabe: mindestens 5 Aufnahmen von Mahlzeiten) variiert beträchtlich. Zum eingeschränkten Zusammenhang zwischen familialer Gesprächsquantität und -qualität vgl. Morek (2012: 96f.); Heller (2012: 108ff). Die Varianzen in der Länge der Cliquen-Interaktionen ergeben sich v. a. aufgrund der unterschiedlichen Lieferzeiten der Pizzadienste und der unterschiedlichen Ausdehnung der Tischgespräche, bis die Kinder von sich aus das ,Ende“ ihres Essens mitteilten.

$8 \mathrm{Zu}$ methodischen Schwierigkeiten beim Kodieren diskursiver Gattungen, die sich v. a. aufgrund intensiver kontextueller Einbettungsverhältnisse ergeben vgl. Morek (2016a). 
richtssequenzen) für die gesprächsanalytische Rekonstruktion ausgewählt (pro Fokuskind je nach Datenlage zwischen 30-70 Sequenzen). Die Ausschnitte wurden nach GAT2 (Selting et al. 2009) transkribiert.

Eine besondere analytische Herausforderung bestand darin, dass die im Material vorfindlichen Sequenzen - stärker als in Elizitationssettings - inhaltlich und interaktiv jeweils höchst heterogen waren. Um sie trotzdem methodisch miteinander in Bezug setzen zu können, wurde das GLOBE-Modell (Hausendorf \& Quasthoff 1996) genutzt. Es wurde für die Beschreibung von narrativen Diskursaktivitäten entwickelt und für Erklärungen (Morek 2012) und Argumentationen (Heller 2012) erweitert. Den Grundannahmen der Interaktionalen Diskursanalyse folgend (vgl. Quasthoff, Heller \& Morek 2017) werden Diskursaktivitäten dabei auf drei miteinander funktional verbundenen Ebenen beschrieben: auf der Ebene der gemeinsam zu bearbeitenden gesprächsorganisatorischen Aufgaben (Jobs), auf der Ebene der inhaltlichen und pragmatischen Züge (Mittel), die die einzelnen Beteiligten liefern, und schließlich auf der Ebene der konkreten sprachlichen Formulierungen (Formen) einschließlich nicht-sprachlicher Formen. Dieser Analysezugang ermöglicht es, Varianzen im je konkreten Vollzug entsprechender Diskursaktivitäten systematisch miteinander zu vergleichen und dabei auch die jeweiligen Anteile der Beteiligten zu extrahieren (vgl. dazu auch Quasthoff \& Kluger in diesem Band).

\section{Ergebnisse im Überblick: Familien- und Peer-Interaktionen im Vergleich}

Grundsätzlich lassen sich anhand der beiden Korpora für die sozialen Veranstaltungen ,familiales Tischgespräch' bzw. ,Cliquenunterhaltung' einige familienbzw. gruppenübergreifende Gemeinsamkeiten beobachten, die offensichtlich jeweils die thematische und kommunikative Typik der entsprechenden Veranstaltungen ausmachen. So kristallisieren sich in den untersuchten Eltern-Kind-Interaktionen folgende wiederkehrende Inhalte heraus: Neuigkeiten und Ereignisse aus Schule, Freundeskreis, Verwandt- und Nachbarschaft; Freizeitaktivitäten und Alltagsorganisation der Familienmitglieder (z. B. Hobbys, Hausarbeit); mittel- und längerfristige Pläne für die Zukunft (z. B. Anschaffungen, Berufe); das körperliche Ergehen der Kinder (z. B. Krankheit, Zahnarzt, Schlaf). Die typischen Themen der Cliquen-Interaktionen sind erwartungsgemäß sehr viel stärker jugendkulturell geprägt: Dort geht es immer wieder um Personen und Vorkommnisse aus Schule und Familie (z. B. Klassenkameraden, Eltern, Lehrer), Medienerfahrun- 
gen (z. B. PC-Spiele, Musik), körperbezogene Themen (z. B. Aussehen, Tabuthemen wie etwa ,Ausscheidungen`) sowie die unmittelbare Gesprächssituation und -umgebung (z. B. Essen, Gegenstände im Raum). ${ }^{9}$

Zwischen diesen thematischen Dominanzen und den jeweils verbreitet auftretenden sprachlich-kommunikativen Praktiken zeigen sich Zusammenhänge (vgl. dazu auch Heller 2012: 108 ff.). So spielt in vielen Familien das Planen anstehender Aktivitäten und das Berichten und Erzählen vom Tag eine zentrale Rolle (vgl. auch Blum-Kulka 1997), während in den Cliquen Praktiken wie z. B. szenisches Erzählen, Dissen und Frotzeln sowie verschiedenste performanceorientierte Aktivitäten (z. B. Sprüche und Zitationen, Rappen, Singen) besonders prominent sind (vgl. dazu z. B. auch Morek 2014; Schmidt 2004; Walther 2014). Darin dokumentieren sich jeweils übergeordnete kommunikative Zwecke der sozialen Veranstaltungen: Am Familientisch geht es sehr wesentlich um das Koordinieren und Teilen des gemeinsamen Alltags sowie um die (Re)Produktion eines gemeinsamen Deutungshaushalts (vgl. Keppler 1994); das doing peer-group (Schmidt 2004) im Rahmen des gemeinsamen Zeitvertreibs dagegen ist auf die Herstellung von Gruppenidentität und Status innerhalb der Gruppe gerichtet und über weite Teile an dem Bemühen um Spaß und wechselseitige Unterhaltung orientiert.

Allerdings fördert der Vergleich zwischen Familien und zwischen Cliquen zutage, dass diese übergeordneten Zwecke gemäß der jeweiligen individuellen, gruppenspezifischen Kommunikationskultur unterschiedlich realisiert werden. Dies wird in Abschnitt 5 detailliert anhand eines Fallvergleichs konkretisiert. Überblicksartig lassen sich die Varianzen in Anlehnung an Heller (2012) zunächst grob in Form von „Gattungsrepertoires“ abbilden. Für die untersuchten Familien ergibt sich das in Tab. 2 dokumentierte Bild.

Im Vergleich bestätigt sich der Befund Hellers (2012), dass Familien in ihren Tischgesprächen offenbar unterschiedlich breite Repertoires an sprachlichdiskursiven Aktivitäten aktualisieren. Es wird die Tendenz sichtbar, dass die Repertoires in sozial privilegierten Familien eher breit und in sozial benachteiligten schmaler sind. Zugleich aber zeigt sich, dass das soziale Milieu allein nicht über die Vielfältigkeit kommunikativer Praktiken entscheidet (vgl. z. B. die Familien von 22w und $44 \mathrm{~m}$ sowie $37 \mathrm{w}$ ). Entsprechendes tritt auch mit Blick auf die fokus-

9 Auch die Familien nehmen bei Tisch Bezug auf Faktoren der unmittelbaren Essenssituation, jedoch nutzen sie diese Ressource (z. B. Anreichen von Speisen) im Vergleich meist seltener und instrumenteller. 


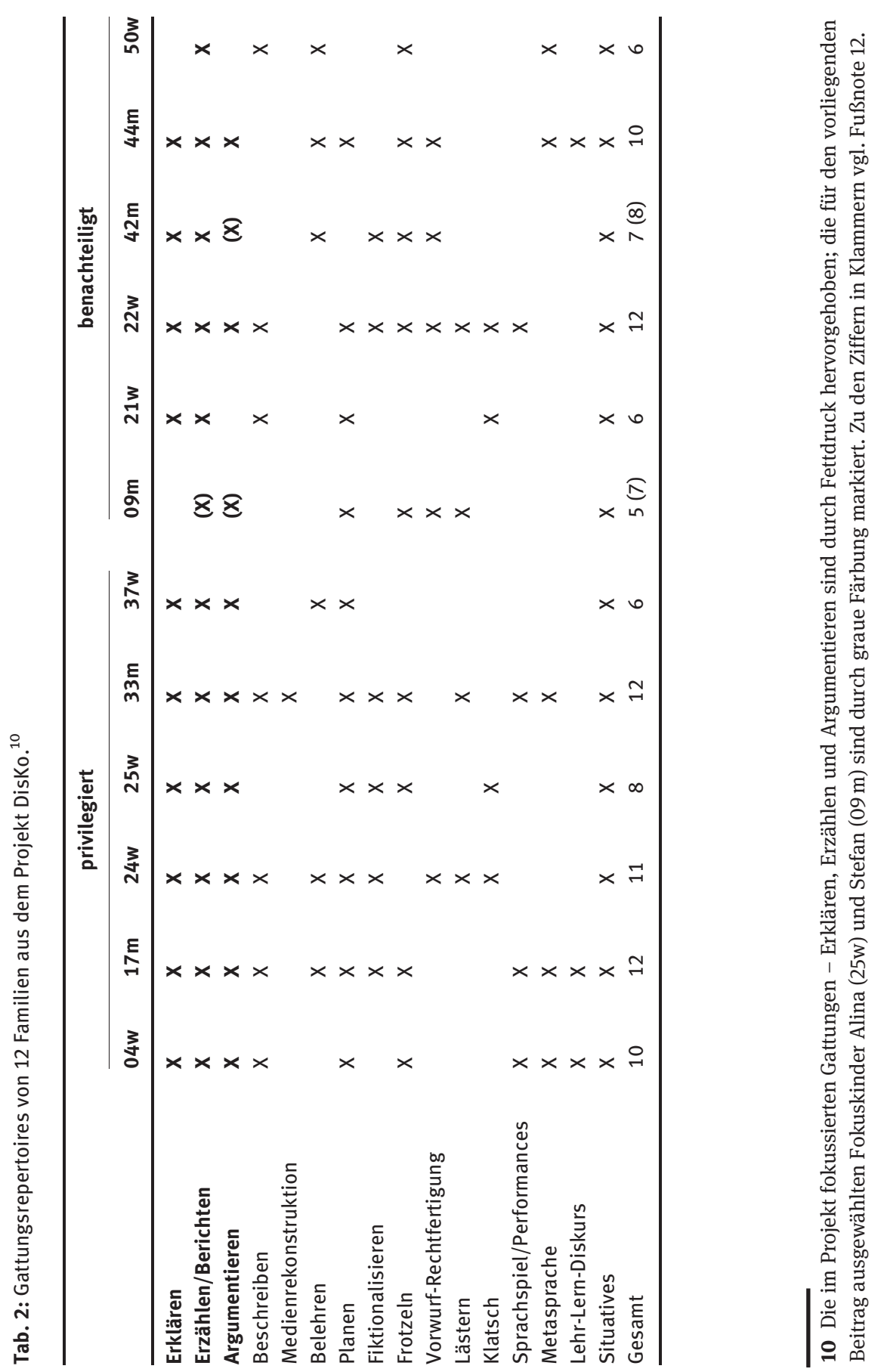


sierten, unterrichtsrelevanten und global strukturierten Gattungen des Erklärens, Erzählens und Argumentierens zutage (vgl. Zellen 1-3): Während diese in den Aufzeichnungen sämtlicher sozial privilegierten Familien des Korpus beobachtbar sind, fehlen sie in einigen der benachteiligten Familien (vgl. auch Morek 2012 zum Erklären; Heller 2012 zum Argumentieren). Dies ist kompatibel mit der auch von Quasthoff et al. (2015: 221) festgestellten, vergleichsweise größeren Varianz in den argumentativen Praktiken sozial weniger privilegierter Familien.

Die auf Kodierungen basierenden Gattungsrepertoires erlauben allerdings weder Aussagen darüber, wie entsprechende Sequenzen von den Beteiligten interaktiv vollzogen werden (z. B. ob und in welcher Weise Kinder überhaupt als Sprecherinnen und Sprecher an den angeführten Aktivitäten teilhaben), ${ }^{11}$ noch darüber, wie häufig die Beteiligten einzelne Gattungen instanziieren. Die Prominenz bestimmter Gattungen lässt sich aber näherungsweise ermitteln, indem man bestimmt, wieviel Prozent der Interaktionszeit für bestimmte Gattungen aufgewendet wird. Eine solche Berechnung wurde für die relativ standardisiert und vom Umfang her vergleichbaren Cliquen-Interaktionen (Wartezeit und Pizza-Essen) vorgenommen. ${ }^{12}$ Tabelle 3 bündelt die Ergebnisse für diejenigen neun Cliquen, aus denen im Projekt Fokuskinder für die kontextvergleichenden Analysen ausgewählt wurden.

Zwei Befunde seien an dieser Stelle hervorgehoben, die unter Gesichtspunkten des außerschulischen Diskurserwerbs besonders relevant sind:

Erstens finden sich zwar in allen untersuchten Cliquen narrative Sequenzen, doch wird an deren unterschiedlichen Anteilen an der Gesamtinteraktionszeit deutlich, dass dem Erzählen z. T. offenbar nur randständiger Status im Gattungsrepertoire einer Gruppe zukommt. Clique C6w etwa verbringt fast die Hälfte der Zeit mit narrativen Aktivitäten (dazu zählen neben dem Erzählen persönlicher Erlebnisse auch das Erfinden fiktiver Geschichten und das Erzählen von Witzen), während in einer anderen Mädchenclique (C5w) der Anteil des Erzählens an der Gesamtinteraktionszeit nicht einmal $3 \%$ ausmacht. Insgesamt sind dabei deutliche milieuspezifische Tendenzen erkennbar, insofern in allen

11 Dies ist auch der Grund, warum das Auftreten narrativer und argumentativer Sequenzen an einigen Stellen mit Klammern versehen ist - die Mikroanalyse der entsprechenden ,candidate sequences‘ (Jefferson 1988: 419) fördert nämlich zutage, dass jeweils nicht i. e.S. etwas narrativ rekonstruiert bzw. argumentiert wird.

12 Der Umfang des Datenmaterials, das die Familien selbst aufgezeichnet haben, variiert sehr stark (zwischen einer und fünf Stunden pro Familie), sodass hier Berechnungen zu Gattungsanteilen an der Interaktionszeit nicht sinnvoll möglich sind. 


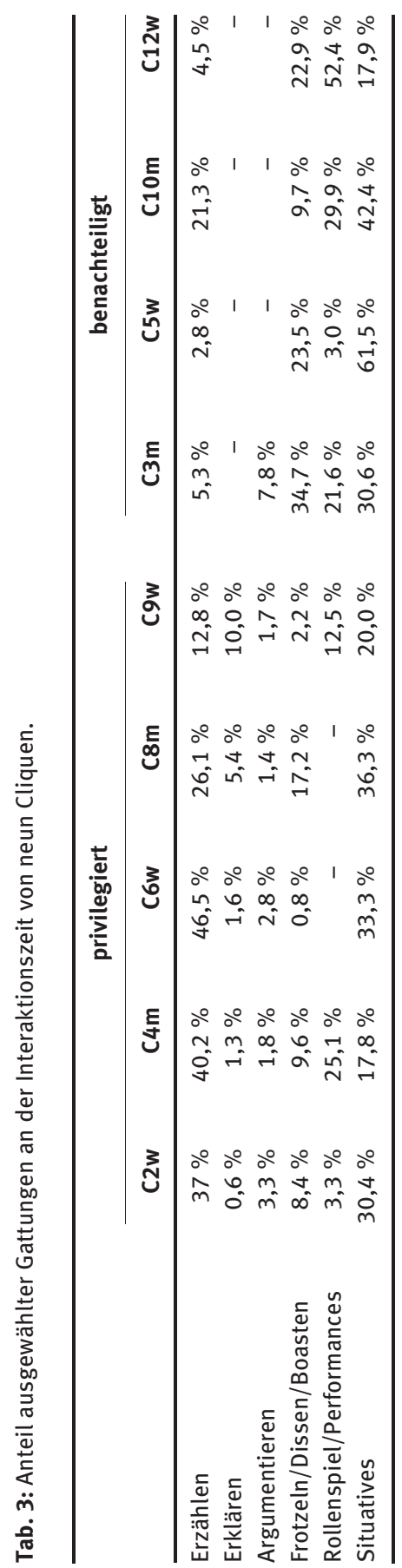


sozial privilegierten Cliquen deutlich mehr Zeit für Erzählen aufgewendet wird als in den sozial benachteiligten.

Zweitens fallen ebenfalls milieuabhängige Disparitäten bei den explanativen und argumentativen Sequenzen ins Auge: So findet sich in keiner der Cliquen aus sozial benachteiligten Kindern Erklären als Praktik, während es unter allen sozial privilegierten Kindern - mindestens kursorisch - dokumentiert ist. Recht vergleichbar gestaltet sich das Bild für argumentative Sequenzen. ${ }^{13}$

Betrachtet man nun zusammenfassend die für die global organisierten narrativen, explanativen und argumentativen Diskurspraktiken verwendete Interaktionszeit im Gesamt, zeichnen sich deutliche Milieuunterschiede ab:

Tab. 4: Anteil narrativer, erklärender und argumentativer Interaktionszeit in 9 Cliquen.

\begin{tabular}{|c|c|c|c|c|c|c|c|c|c|}
\hline & \multicolumn{5}{|c|}{ privilegiert } & \multicolumn{4}{|c|}{ benachteiligt } \\
\hline & $\mathrm{C} 2 \mathrm{w}$ & $\mathrm{C} 4 \mathrm{~m}$ & C6w & $\mathrm{C} 8 \mathrm{~m}$ & C9w & C3m & C5w & $\mathrm{C} 10 \mathrm{~m}$ & $\mathrm{C} 12 \mathrm{w}$ \\
\hline$E R Z$ + ERK + ARG & $40,9 \%$ & $43,3 \%$ & $50,9 \%$ & $32,9 \%$ & $24,5 \%$ & $13,1 \%$ & $2,8 \%$ & $21,3 \%$ & $4,5 \%$ \\
\hline
\end{tabular}

Während in den sozial privilegierten Cliquen die betreffenden global organisierten Praktiken etwa ein Viertel bis die Hälfte der gemeinsamen Interaktionszeit ausmachen, beträgt der entsprechende Anteil unter den sozial benachteiligten Präadoleszenten in allen Fällen deutlich unter einem Viertel. In diesen Cliquen stehen die überwiegende Zeit Praktiken im Vordergrund, die lokal organisiert und stark situationsgebunden sind (vgl. auch Morek 2014), wie z. B. rollenspielähnliche verbale Schlagabtäusche, ,Sprüche-Klopfen` und/oder (z. B. scherzhafte) Kommentare zum unmittelbaren gemeinsamen Handlungs- und Wahrnehmungskontext (vgl. auch Tab. 3).

Insgesamt also, so legt die überblicksartige Auswertung der Gattungsrepertoires nahe, unterscheiden sich die untersuchten Cliquen und Familien deutlich darin, welche kommunikativen Praktiken sie in der gemeinsamen sozialen Interaktion relevantsetzen und wie prominent jeweils global strukturierte Diskurspraktiken

13 Allerdings fällt der - angesichts der übrigen Tendenzen erwartungswidrig - hohe Anteil des Argumentierens in der Clique C3m auf. Er kommt sehr wesentlich dadurch zustande, dass zwei Gruppenmitglieder in einer längeren, von performativen Einsprengseln durchsetzten, Sequenz darüber ,streiten', wer der schnellste Rapper der Welt ist. Die übrigen Gruppenmitglieder haben nicht aktiv Anteil an argumentativen Sequenzen. 
dabei vertreten sind. ${ }^{14}$ Dabei scheinen milieuabhängige Disparitäten bemerkenswerterweise in den Cliquen-Interaktionen noch deutlicher zutage zutreten als in den Familiengesprächen; dies könnte damit zusammenhängen, dass globale Diskursaktivitäten in ihrer großen sequenziellen Reichweite den handlungsleitenden Maximen von Peer-Interaktion, nämlich ,Schnelligkeit‘ und ,Wettbewerb um das Rederecht' widersprechen. Insgesamt scheinen sich gerade für Kinder aus sozial privilegierten Milieus reichhaltige und strukturell auch im Unterricht anschlussfähige Diskursaktivitäten in der Familie mit ebenso reichhaltigen und unterrichtlich nutzbaren Interaktionserfahrungen in der Peergroup zu einem anregungsreiche(re)n außerschulischen Kommunikationsalltag zu addieren. Kinder aus sozial benachteiligten Milieus dagegen scheinen wesentlich stärker auf die Familie allein als Anwendungs- und Erwerbskontext für diskursive Aktivitäten angewiesen zu sein.

Wie unterschiedlich im Einzelfall die alltäglichen Interaktionserfahrungen einzelner Kinder in ihren Familien und Cliquen beschaffen sind und was dies jeweils konkret für Möglichkeiten und Grenzen des kindlichen Diskurserwerbs bedeutet, wird im folgenden Abschnitt 5 anhand eines Fallvergleichs gezeigt. $\mathrm{Zu}$ diesem Zweck werden ausgewählte - argumentative und explanative - Ausschnitte aus Familien- und Peer-Interaktionen zweier Fokuskinder miteinander kontrastiert; sie werden jeweils mit Blick auf interaktive und sprachliche Strukturen ihres konkreten Vollzugs mikroanalytisch untersucht.

\section{Außerschulische Erwerbskonstellationen im exemplarischen Fallvergleich}

\subsection{Die Fokuskinder ,Alina“ und ,Stefan“}

Ausgewählt wurden die hier vorzustellenden Fälle (,Alina‘, 25w und ,Stefan‘, 09m) nach dem Prinzip des größtmöglichen Kontrasts, was a) die Art und Breite ihrer außerschulischen Kommunikationserfahrungen in Familie und Clique betrifft und b) ihre gezeigten Diskurskompetenzen - einschließlich der interaktiv erfolgreichen Partizipation an unterrichtlichen Diskurspraktiken (vgl. dazu Quasthoff \& Morek 2015). Mit Blick auf beide Kriterien repräsentieren diese Kinder jeweils

14 Allerdings muss einschränkend im Blick behalten werden, dass der vorliegenden qualitativen Studie naturgemäß (s. Quasthoff in diesem Band) ein zahlenmäßig begrenzter Datenausschnitt aus Familien- und Peer-Interaktionen vorliegt; zur Generalisierung müssten die Beobachtungen an größeren Korpora statistisch überprüft werden. 
das obere bzw. das untere Ende des im DisKo-Projekt rekonstruierbaren Spektrums: Während Alinas Familien- und Peer-Interaktionen durch Reichhaltigkeit diskursiver, und dabei v. a. auch unterrichtsrelevanter Praktiken gekennzeichnet sind und Alina hohe Diskurskompetenzen v. a. im Bereich der Kontextualisierung zeigt, ergibt sich bei Stefan ein gegenteiliges Bild: Er repräsentiert Kinder mit wenig ausgeprägten Diskurskompetenzen, bei denen sowohl in der Familie als auch in der Peergroup kaum jemals globale Diskurspraktiken kontextuell gefordert werden (vgl. Tab. 2-4).

Tabelle 5 kontrastiert - wiederum gemäß dem Fokus von DisKo auf narrative, argumentative und explanative Diskurspraktiken -, wie viel Prozent der aufgezeichneten Peer- bzw. Familieninteraktionen Alinas und Stefans jeweils für die globalstrukturellen Aktivitäten Erzählen, Argumentieren und Erklären aufgewendet werden; die absolute Zeitdauer (in Minuten) findet sich jeweils zusätzlich in Klammern. ${ }^{15}$

Tab. 5: Anteil narrativer, argumentativer und explanativer Diskursaktivitäten an den Peer- und Familieninteraktionen von Alina und Stefan.

\begin{tabular}{lllllllll}
\hline & \multicolumn{3}{c}{ Peer-Interaktionen } & & \multicolumn{3}{c}{ Familieninteraktionen } \\
\cline { 2 - 3 } \cline { 6 - 8 } & NARR & ARG & ERKLÄ & & NARR & ARG & ERKLÄ \\
\hline Alina $(25 \mathrm{w})$ & $46,5 \%$ & $2,8 \%$ & $1,6 \%$ & & $16,6 \%$ & $4,2 \%$ & $4,5 \%$ \\
& $(19: 00 \mathrm{~min})$ & $(01: 20 \mathrm{~min})$ & $(00: 45 \mathrm{~min})$ & $(34: 20 \mathrm{~min})$ & $(08: 45 \mathrm{~min})$ & $(09: 25 \mathrm{~min})$ \\
Stefan $(09 \mathrm{~m})$ & $5,3 \%$ & $7,8 \%$ & - & & $0,7 \%$ & $0,8 \%$ & - \\
& $(01: 10 \mathrm{~min})$ & $(02: 15 \mathrm{~min})$ & & & $(00: 25 \mathrm{~min})$ & $(00: 30 \mathrm{~min})$ & \\
\hline
\end{tabular}

Im direkten Vergleich der beiden Fokuskinder zeigt sich, dass rund die Hälfte der Zeit in Alinas Peer-Interaktionen auf narrative, argumentative und explanative Diskursaktivitäten verwendet wird. In Stefans Clique sind es nur ca. 13 \%, wobei sich Erklären i. e. S. überhaupt nicht findet (vgl. aber 5.2.2). Stefan ist weder an den vorfindbaren Erzähl- noch Argumentationssequenzen als Sprecher beteiligt. Noch deutlicher fallen die Unterschiede im Vergleich der beiden Familien aus: Alinas Familie wendet rund ein Viertel der Zeit ihrer Tischgespräche für narrative, argumentative und explanative Diskursaktivitäten auf; bei Stefan sind es lediglich 1,5 \% und wiederum finden sich keinerlei Erklärsequenzen. Den Hauptanteil sowohl in seinen Peer- als auch in seinen Familieninteraktionen nehmen unmittelbar

15 Bei der absoluten Zeitdauer ist insbesondere im Fall der Familieninteraktionen zu berücksichtigen, dass sich die jeweils aufgezeichneten Gesprächsdaten in ihrem Gesamtumfang stark unterscheiden (vgl. dazu Tab. 1). 
situationsgebundene, lokal-sequenziell organisierte Aktivitäten ein (Essensorganisation, Kommentare und thematische Interaktion zu situativ Wahrnehmbarem, in der Clique darüber hinaus: kurze spielerische Inszenierungen, Zitationen und andere - auch rein körperliche - Performances, z. B. „Spaßkämpfchen'). Das Hierund-Jetzt der gemeinsamen Essenssituation übersteigende thematische Interaktion (vgl. dazu auch Heller 2012: 109f.) ist selten. ${ }^{16}$ Insgesamt repräsentiert Stefans Familie den mit Abstand ,anregungsärmsten' familialen Interaktionskontext des Korpus.

Zum Zeitpunkt des Erhebungsbeginns sind beide Kinder elf Jahre alt, leben als Einzelkinder mit jeweils beiden Elternteilen zusammen und sprechen Deutsch als Mutter- und einzige Familiensprache. Alina besucht die fünfte Klasse eines Gymnasiums mit sozial privilegierter Schülerschaft; beide Eltern sind berufstätig (Vater: Elektroingenieur, Mutter: Versicherungsangestellte). Stefan besucht die fünfte Klasse einer Hauptschule; seine Mutter ist Hausfrau, sein Vater Arbeiter in einer Fabrik. ${ }^{17}$ Alina und Stefan bilden also nicht nur mit Blick auf ihre diskursiven Erfahrungen und Kompetenzen, sondern auch hinsichtlich ihres sozialen Herkunftsmilieus komplementäre Fälle.

Für beide Kinder wird im Folgenden jeweils eine Sequenz aus der Familiensowie eine aus der Peer-Interaktion vorgestellt. Die daran exemplarisch aufzuzeigenden Analyseergebnisse adressieren die in 2.3 entwickelten Fragen:

1a. Wie ist der Ausschnitt vor dem Hintergrund des Gattungsrepertoires der betreffenden Interaktionsgemeinschaft einzuordnen?

1b. Welche Interaktionsmuster werden vollzogen?

1c. Welche Realisierungen diskursiver Strukturen zeigen sich auf Kontextualisierungs-, Vertextungs- und Markierungsebene?

2. Wie trägt das jeweilige Fokuskind mit seinen Äußerungen zur interaktiven Hervorbringung des entsprechenden sequenziellen Kontexts bei?

\subsection{Familieninteraktionen Alinas und Stefans}

Für den exemplarischen Vergleich der Familieninteraktionen von Alina und Stefan wurden zunächst zwei gut parallelisierbare Sequenzen ausgewählt, die

16 Am Beispiel von Stefans Familieninteraktion zeigen sich die erwähnten Zusammenhänge zwischen der Dauer der jeweiligen Gespräche und ihrer thematischen und diskursiven Tiefe: Die aufgezeichneten Tischgespräche dauern bei Stefan durchschnittlich nur zehn Minuten und sind damit die kürzesten im vorliegenden Korpus.

17 Das Item ,Bücherbestand im Haushalt‘ aus dem Fragebogen untermauert die Unterschiede im sozio-kulturellen Milieu (Alina: mehr als 200 Bücher (4. Stufe auf vierstufiger Skala), Stefan: 26-100 Bücher (2. Stufe auf vierstufiger Skala)). 
beide ihren Ausgangspunkt in der Tages- bzw. Wochenendplanung haben und damit einem besonders familientypischen Thema (Heath 2006) entspringen. Da es jeweils um eine Entscheidungsfindung geht, bringen beide Ausschnitte argumentatives Potenzial mit sich.

In Alinas Tischgesprächen nimmt das Argumentieren einen festen Platz ein und findet sich i.d.R. als konsensuelles Argumentieren im von Kotthoff (2015: 83f.) beschriebenen „Räsoniermodus“. Dieser ist dadurch gekennzeichnet, dass die Beteiligten potenziellen Dissens antizipieren, proaktiv Begründungen liefern und abwägen, und sich wechselseitig aufzeigen, dass sie grundsätzlich ähnliche Positionen zur in Frage stehenden Quaestio (Klein 1980) einnehmen.

In Stefans Familie finden sich, wie bereits skizziert, so gut wie keine globalen Diskursaktivitäten (vgl. Tab. 2). Zwar lassen sich durchaus mehrere Passagen identifizieren, wo (lokale) Begründungen angebracht werden. Dies geschieht jedoch i.d.R. im Modus des einseitigen Begründens (vgl. Heller 2012: 140), und zwar v. a. in Form von Rechtfertigungen in Reaktion auf Vorwürfe.

Vor diesem Hintergrund repräsentiert der Ausschnitt „Judo“ (Beispiel 1) für Alinas Familie einen typischen Fall. Der Ausschnitt „Geburtstagsgeschenk“ (Beispiel 2) hingegen stellt für Stefans Familieninteraktionen einen Sonderfall dar, insofern er einen kindlichen Versuch enthält, Argumentieren i. S. des wechselseitigen Austausches von Positionen und Begründungen zu initiieren.

\subsubsection{Alinas Familieninteraktion: Das Beispiel exploratives Argumentieren im Räsoniermodus}

Im folgenden Ausschnitt sprechen Alina und ihre Mutter über das Hobby der Tochter, Judo. Es geht um die Frage, ob Alina ,judo vielleicht AUFhör[n]“ (Z. 21) soll.

(1) Judo (25w-C6w-Gy2-F2), Mu: Mutter, Al: Alina ${ }^{18}$

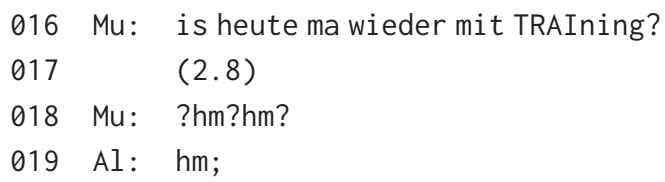

18 Alle Transkripte wurden gemäß GAT 2 (Selting et al. 2009) erstellt. Die entsprechenden Konventionen finden sich im Anhang dieses Bandes sowie unter http://www.gespraechsfor schung-ozs.de/heft2009/px-gat2.pdf (letzter Zugriff: 17.09.2020). 
020

021

022

02

024

025

026

027

028

029

030

031

032

033

034

035

036

037

03

03

04

04

04

043

044

045

046

047

(. . .)

061 Mu: vielleicht denkst_e da mal noch n_bisschen drüber NACH;

$062 \quad(-)$ wo du SPAß dran hättest;

063 Al: hm_hm,

$064 \mathrm{Mu}$ : kannst ja verschiedene dinge mal ANschauen,

$065 \quad(1.1)$

066 ne ich glaub KüNdigen können wir sowieso erst zum ENde des jahres.

$067 \mathrm{Al}: \mathrm{OH} ;=\mathrm{HM}$. 
068 is ja noch LANge zeit.

069 Mu: jaja EBen.

070 (9.2)

Kennzeichnend für argumentative Diskursaktivitäten ist die sequenzielle Bearbeitung folgender Gesprächsaufgaben (Heller 2012: 1) ,Konstituieren von Dissens‘ bzw. ,Problematisierung von Geltungsansprüchen‘, 2) ,Etablieren von Begründungspflicht‘, 3) ,Austausch von Begründungen“, 4) ,Abschließen der Argumentation'.

Betrachtet man nun, wie sich Mutter und Kind jeweils in die Bearbeitung dieser Jobs einbringen, zeigt sich aufseiten Alinas ein hoher Eigenanteil, gepaart mit fordernd-unterstützenden (vgl. 2.2) Zuhöraktivitäten der Mutter. Zunächst greift Alina die thematische Relevantsetzung der Mutter (,,is heute ma wieder mit TRAIning?“, Z. 16) auf und wirft das Einstellen des Hobbys als offene Frage auf (,ich überLEG mittlerweile schon (-) ob ich judo vielleicht AUFhör;“, Z. 21). Damit nimmt sie selbst eine Problematisierung vor - doch nicht nur das: Sie instanziiert auch selbst Begründungpflicht, indem sie unmittelbar eine Begründung anschließt (,weil es macht mir ja schon LANge (.) keinen spass mehr;“, Z. 22). Die Konklusion, das Hobby zum vorliegenden Zeitpunkt - aus momentan empfundener Unlust - gleich vollständig ad acta zu legen, wird jedoch von der Mutter mit divergenzanzeigenden Nachfragen als nicht tragfähig behandelt („EHRli:ch,“, Z. 23, „möchts_du GANZ aufhörn;“, Z. 24). Nach kurzem Überlegen (Z. 25-27) wiederholt Alina daraufhin ihr Argument, allerdings inhaltlich anders akzentuiert (,ich geh ja no_ni_mals gern zum TRAIning;“, Z. 29). Nachdem die Mutter schweigt (Z. 30) und somit den Zugzwang zum Ausbau der Begründung aufrechterhält, ${ }^{19}$ baut Alina ihren Turn zu einem längeren, argumentativen „Äußerungspaket“ aus (Morek, Heller \& Quasthoff 2017): Mit dem Verweis auf das Fehlen einer gewichtsmäßig (Z. 31-33) bzw. vom Leistungsniveau her (Z. 36-39) passenden Trainingspartnerin führt sie nun sachliche Ursachen für ihre mangelnde Trainingsfreude ins Feld. Der Wechsel von einer rein subjektiv-affektbezogenen zu einer stärker objektivierten Begründung wird von zustimmenden und affiliativen Hörersignalen der Mutter begleitet (Z. 34, 38). Durch diese Continuer - und Schweigen (Z. 35) - wird die argumentative Elaborierung Alinas sowohl ratifiziert als auch eingefordert.

In Zeile 40 folgt dann ein Einwand der Mutter gegen Alinas Argument der Niveauunterschiede (,ja gut aber das WÄR doch schon mal was-“). In verfrühter

19 Dies wiederholt sich in Z. 35. Auch dort wird der Zugzwang zum Begründen für Alina aufrechterhalten. 
Reaktion darauf bezieht Alina diesen lediglich auf die zuvor behauptete Gewichtsgleichheit zwischen sich selbst und Lisa; sie fügt ein scherzhaft modalisiertes, präzisierendes Zugeständnis an (,okay sie is «:-) zwei kilo LEICHter aber-“, Z. 41). Auf das eigentliche Gegenargument der Mutter (Z. 42), dass sich Niveauunterschiede doch mit der Zeit nivellierten, reagiert Alina nicht mehr. An der Reaktion der Mutter (Z. 43ff.) wird jedoch sichtbar, dass die Beteiligten das Begründen nun als beendet behandeln und den Abschluss der Quaestio-Bearbeitung einläuten: Die Mutter macht einen Kompromissvorschlag (,Teilnahme nur am Training, nicht aber an Turnieren‘, Z. 43-44), stellt Alina die Suche nach möglichen Alternativhobbys anheim (Z. 47, Z. 61-62 und Z. 64) und thematisiert eine mögliche Kündigung des Judo-Trainings (Z. 66). Das Abschließen der Argumentation wird hier also sehr wesentlich von den verschiedentlichen Konvergenzangeboten der Mutter strukturiert und dabei interaktiv von beiden Beteiligten vollzogen. Die letztliche Entscheidung wird einvernehmlich vertagt (Z. 67-70).

Auf Ebene der argumentationsstrukturierenden Jobs zeigt sich also insgesamt, dass Alina bei der anfänglichen Lancierung des Argumentierens sehr eigenständig agiert und im Verlauf der Sequenz, v. a. beim eigentlichen Begründen sowie im Abschluss, durch Gesprächsaktivitäten der Mutter interaktive Hilfestellung erfährt: problematisierende Nachfragen (Z. 23-24), Continuer (Z. 26, 34, 38), sequenziell spezifisch platziertes Schweigen (Z. 27, 35) sowie Einwände (Z. 40) sind hier Indizien des elternseitigen Forderns und Unterstützens. Dass sich dieses Interaktionsmuster dabei teilweise recht subtil zeigt (z. B. Schweigen, Continuer), dürfte zum einen direkter interaktiver Reflex von Alinas eigenständiger Kontextualisierung und damit ihres Kompetenzniveaus (vgl. Quasthoff \& Kluger in diesem Band) sein; zum anderen ist eine solche defensive Interaktionsweise auch plausibel angesichts des Modus des konsensorientierten, explorativ und räsonierend angelegten Argumentierens.

Durch die fordernd-unterstützenden Gesprächsaktivitäten der Mutter auf Mittelebene werden nun - erwerbstheoretisch betrachtet - fortwährend argumentative Anforderungen an Alina etabliert, die v. a. in einem Ausbau auf Ebene der Vertextung resultieren: Die zunächst angebotene subjektiv-assoziative Begründung, die für frühe Stadien des Argumentationserwerbs kennzeichnend ist (z. B. Grundler \& Vogt 2013; Pohl 2014), wird im weiteren Gesprächsverlauf gestützt durch eine sachlichere, objektivierte Begründung, die auf die Entkräftung von (potenziellem) Widerspruch gerichtet ist. Auch strukturell betrachtet erweist sich die zweite, in Reaktion auf die elterlichen Rückmeldungen gelieferte Begründung (Z. 31-39) als komplex, insofern sie zwei Gründe miteinander koppelt (,keine passende Gewichtsklasse‘, ,kein passendes Leistungsniveau') und diese implizit konzessiv miteinander verbindet („Anouk (-) ist nicht mehr auf meinem niVEAU,“, Z. 36; 
„aber halt in meinem geWICHT ungefähr.“, Z. 39). Damit wird nicht nur der mögliche Einwand bearbeitet, dass durchaus gleichgewichtige Trainingspartnerinnen zur Verfügung stünden. Durch die Entfaltung einer Dilemma-Konstellation fügen sich die beiden Argumente auch auf höherer Ebene zu einem übergeordneten Argument (,kein geeigneter Trainingspartner'). Ferner ist mit Blick auf Alinas Vertextung $\mathrm{zu}$ beobachten, dass sie a) eine als Beleg fungierende Illustrierung einschiebt („mona lara guiselle- (-) sind alle SCHWErer,“, Z. 33) sowie eine ebenfalls dialogisch auf die Interaktionspartnerin zugeschnittene eingeschobene Erläuterung der Prämisse, dass die von der Gewichtsklasse her passende Freundin leistungsmäßig zurückliegt (,weil sie so lange (-) nicht geMACHT hat,“, Z. 37).

Diese komplexe Vertextung Alinas enthält zahlreiche sprachliche Formen, die die inhaltliche Struktur und die Rahmung des Ganzen als konsensuelles, exploratives Argumentieren markieren. Einige seien hier erwähnt: Neben kausalen (weil, Z. 22, 28, 37), additiven (auch, Z. 31), adversativen (aber, Z. 39, 41) und konzessiven Konnektoren (naja, Z. 31) fällt vor allem der rege Gebrauch der Modalpartikel ja auf (Z. 22, 28, 29, 31), die - wie auch die Formulierung „du siehst es ja SELBST“ (Z. 28) - besonders geeignet ist, um die angeführten Gründe als „kollektiv Geltendes“ (Klein 1980) zu rahmen. Eingangs eingesetzte Formen wie das Modalwort vielleicht sowie die Konstruktion aus Matrixsatz mit Verbum cogitandi überlegen und subordinierter, deliberativer ob-Frage (Weinrich et al. 2007: 881) (Z. 21) erfüllen darüber hinaus die Funktion, den explorativen, ergebnisoffenen Charakter der Argumentation zu unterstreichen (vgl. auch Morek 2020).

Zieht man mit Blick auf die Betrachtungsebenen der Vertextung und Markierung auch die Äußerungen der Mutter hinzu, so zeigt das Beispiel, dass auch sie dialogisch-konzessive Strukturen etabliert (Z. 40) und darüber hinaus - unter Nutzung des Konjunktiv II - Kompromisse und Alternativoptionen eröffnet (Z. 40, 47, 62). Insgesamt aktualisieren Mutter und Tochter auf diese Weise ein breites Repertoire an Mitteln der argumentativen Vertextung sowie deren sprachlich-formaler Umsetzung, das anschlussfähig sein dürfte auch für konsensorientiertes, exploratives Argumentieren etwa in unterrichtlichen Kontexten. Der obige Ausschnitt dokumentiert, dass Alina mit ihren familialen Interaktionen ein reichhaltiger, anregungsreicher Kontext für Erwerb und Ausbau schulisch relevanter diskursiver Kompetenzen zur Verfügung steht.

Zugleich lässt sich (vgl. Analysefrage 2, 2.3) am obigen Ausschnitt auch zeigen, wie interne und externe Ressourcen des Diskurserwerbs zusammenspielen: Mikroanalytisch wird rekonstruierbar, dass und wie der entsprechende sequenzielle Kontext - eine spezifisch gerahmte argumentative Sequenz im Rahmen eines familialen Tischgesprächs - in beträchtlichem Maße auch durch kindseitige Gesprächsaktivitäten hervorgebracht wird. Dass es nämlich über- 
haupt zu einer interaktiv vollzogenen, elternseitig unterstützten Argumentationsaktivität kommt, wird wesentlich von Alina mit vorbereitet.

Als Stellschraube des interaktiven Erfolgs bei ihrer recht eigeninitiativen Lancierung einer Diskursaktivität erweist sich deren sequenzielle Kontextualisierung (Morek \& Quasthoff 2018): So knüpft Alina thematisch unmittelbar an den zuvor etablierten Fokus an (,zum Training gehen' - ,Judo aufhören', Z. 16 und Z. 21) und projiziert erkennbar den Eintritt ins Argumentieren, indem sie sowohl eine Problematisierung (Z. 21) als auch die Instanziierung des Begründens (Z. 22) leistet. Den darauffolgenden impliziten Zugzwang in die Gattung hinein - die erstaunte Rückfrage der Mutter - erkennt und bedient sie. Zudem leistet Alina auch unter Gesichtspunkten der sozialen Kontextualisierung einen Zuschnitt auf die Zuhörerin bzw. die gemeinsame Kommunikationskultur: Sie greift nicht nur eine elternseitige Relevantsetzung auf, sondern wählt v. a. mit dem konsensorientierten Argumentieren im Räsoniermodus eine für das Gattungsrepertoire dieser Familie zentrale Praktik, die sie der Mutter deutlich erkennbar macht: Das Problematisieren wird als Resultat eines bereits länger andauernden und ergebnisoffenen Reflexionsprozesses gerahmt, und zwar z. B. durch zahlreiche temporal und epistemisch modalisierende Ausdrücke (z. B. mittlerweile, schon lange, vielleicht, ich überleg, ich weiß nicht). Auf diese Weise treten Mutter und Tochter in ein wechselseitiges, räsonierendes Argumentieren ein (vgl. Heller 2018; Morek 2020).

Betrachten wir nun demgegenüber die Familieninteraktion des Fokuskindes Stefan.

\subsubsection{Stefans Familieninteraktion: Diskursarmut und Kontextualisierungsinkonsistenz}

Im folgenden Ausschnitt aus Stefans Familie geht es inhaltlich um die Frage, was einem befreundeten Jungen bei der anstehenden Geburtstagsfeier geschenkt werden soll (ab Z. 16). Zuvor wird - ausgehend von einem elternseitigen Vorwurf (Z. 2) - der Zeitpunkt der Erledigung von Stefans Hausaufgaben verhandelt (Z. 3-7) und das Essen kommentiert (Z. 12-13).

(2) Geburtstagsgeschenk (09 m-C3m-H1-F1), Va: Vater, St: Stefan, Mu: Mutter

002 Va: du erzÄHLST doch immer du hast nix AUF.

003 St: HEUT aber.

004 Va: dat kannste auch heu' MORgen machen.

$005 \quad(0.7)$ 
$006 \mathrm{Mu}:$ morgen is SAMStag;

007 St: n_JA.

$008 \quad(1.1)$

009 Va: ((stöhnt langgezogen))

$010 \quad(11.0)$

011 ((Rascheln einer Brötchentüte o.ä. ))

012 Va: is AU leer; =ne?=

$013=($ das $/$ der $)$ schmeckt GU: : :T.

$014 \quad(1.0)$

$015 \mathrm{Mu}: \quad[(\quad)]$

016 St: [was soll ] ich (dem/ihm) denn GE: : ben;

$017 \quad(0.9)$

$018<<p>$ zum geBURTStagsgeSCHENK; >=

$019 \mathrm{Mu}: \quad=<<$ stöhnend $>$ wei : : B ich AU_no_ni: : :ch;>

$020 \quad(6.2)$

021 Mu: feiert der franz jetzt MORgen?=

$022 \quad=$ oder überMOR[gen; ]

$023 \mathrm{Va}: \quad[<<$ monoton $>$ am $][$ ! SONN! tag. $>]$

024 St: [soll ich den ] so einen

BALL schenken?

$025 \mathrm{Mu}:$ SONNtag?

026 Va: SONNtach.

$027 \mathrm{Mu}$ : biss_e dir SIcher;

$028 \mathrm{Va}: \mathrm{JA} .=$

029 St: =PApa,_soll ich (ihn) so einen BALL schenken; =

$030 \quad=$ so einen WEIßen da: : ?

$031 \quad(1.5)$

032 St: ich hab doch S0 [einen-]

$033 \mathrm{Va}$ [ [hat_er] bestimmt!SEL!ber sich ge (.) geholt;

034 St: $<<t$, dim $>$ nei:: :,$=$

$035 \quad=$ !BIT! te: : : :;>

036 Va: <<cresc> !NEI!_E: :N;>

$037 \quad(1.7)$

038 St: der HAT keine !BÄL!le; =

$039=$ der hat nur so_n PLAStikball;

$040 \quad(1.0)$

041 und_n e: EM ball; =

$042=$ =mehr au NICH;

$043 \quad$ (6.3)

044 Va: ich !WEIß! es NICH; 


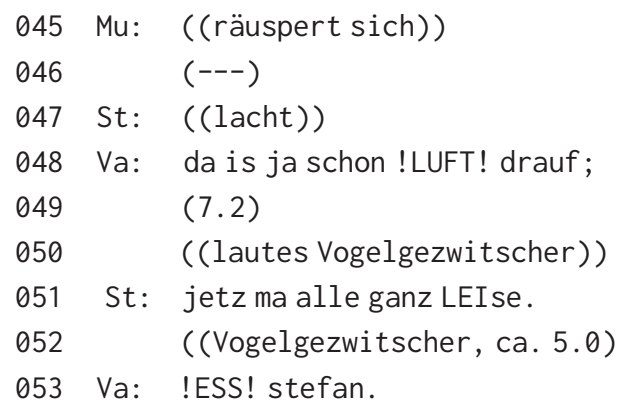

Betrachten wir auch für diesen Ausschnitt zunächst die Frage, wie Eltern und Kind mit den argumentationskonstitutiven Gesprächsaufgaben umgehen. Dabei fällt auf, dass zunächst gar nicht ersichtlich ist - und zwar weder für die/den Analysierende/n noch für die Beteiligten -, dass hier überhaupt Argumentieren als Diskursaktivität relevantgesetzt wird. Denn mit der Frage „was soll ich (dem/ ihm) denn GE::ben;“ (Z. 16) setzt Stefan einen Zugzwang, der auf den Erhalt eines Ratschlags gerichtet ist. Ein Ratschlag formuliert eine nach Meinung des Ratgebers empfehlenswerte Handlungsoption und liefert so dem Ratsuchenden eine mögliche Problemlösung (vgl. Zifonun, Hoffmann \& Strecker 1997: 137). Damit ist Stefans Eingangsfrage einlinig auf den Erhalt einer elternseitigen Einschätzung ausgerichtet. Diesen Zugzwang reformuliert er noch zwei weitere Male (Z. 24, 29), und zwar indem er einen konkreten Geschenkvorschlag zur Disposition stellt („,soll ich den so einen BALL schenken“, Z. 24). Nachdem die Eltern sich zunächst dem Zugzwang entziehen (z. B. „wei::ß ich AU_no_ni:::ch;“, Z. 19), aber die Entscheidungsgewalt für sich reklamieren, weist der Vater in Zeile 33 die kindseitig eingebrachte Option begründet zurück: „hat_er bestimmt !SEL!ber sich ge (.) geholt;“.

Erst daraufhin wird sichtbar, dass sich hier ein potenziell argumentativer Gesprächskontext interaktiv manifestiert: Mit der prosodisch gedehnten Äußerung des Responsivs nein (Z. 34) etabliert Stefan deutlichen Dissens. Allerdings tritt er dennoch nicht ins Argumentieren ein, sondern wählt eine andere Art der Dissensbearbeitung, nämlich Betteln (,!BIT!te::::“, Z. 35). Damit deutet er den vorangegangenen Austausch retrospektiv als eine auf Erlaubniserteilung bzw. -verwehrung angelegte Frage-Antwort-Sequenz um, was auch von dem Vater entsprechend aufgenommen wird. Auf Stefans Bitten hin insistiert der Vater auf seiner Meinung (,,INEI!E::N“, Z. 36) und leitet mit dieser abschlägigen Antwort die potenzielle Beendigung der Sequenz ein.

Im Sinne eines moving-out-of-closing (Button 1987) produziert Stefan dann einen Widerspruch (,der HAT keine !BÄL!le;“, Z. 38), mit der er die Vermutung des Vaters, der Beschenkte sei bereits im Besitz eines Balles (Z. 33) zurückweist. 
Es folgt eine Stützung („der hat nur so_n PLAStikball; [. . .] und_n e: EM ball; mehr au NICH;“, Z. 39-41), die jedoch vom Vater nicht weiter beachtet wird: Wie schon zuvor die Mutter entzieht sich der Vater - mit einer als Account fungierenden no-knowledge-response weiterer Elaborierung (vgl. Clayman 2002: 422-423) (,ich !WEIß! es NICH;“, Z. 44). Eine inhaltliche Reaktion auf Stefans Argument, ein Plastik- und ein EM-Ball seien keine ,richtigen“ (Ventil)Fußbälle, liefert er verzögert nach („da is ja schon !LUFT! drauf;“, Z. 48). Dieses Gegenargument wird nun wiederum von Stefan ignoriert, der nach einer Schweigephase die Aufmerksamkeit auf das am Essplatz zu hörende Vogelgezwitscher (Z. 51) lenkt. Damit wird die Sequenz nicht zu einem wechselseitig ratifizierten (temporären) Konsens geführt, sondern wird stillschweigend fallengelassen, sodass sie versandet (vgl. Heller 2012: 95).

Insgesamt dokumentiert dieser Ausschnitt die argumentationsspezifische Ausprägung des Musters „Dulden und Tilgen“ (Morek 2012: 135ff.), die Heller (2012) schon für einige familiale Tischgespräche rekonstruieren konnte: das „Dulden und Fallenlassen“. Für dieses Muster ist kennzeichnend, dass elternseitig kaum globale Anforderungen an das Kind etabliert werden. Eingeschränkten Gesprächs- und Zuhöraktivitäten der Erwachsenen stehen kindseitige Eigeninitiativen gegenüber, mit denen die Kinder Diskursaktivitäten anzustoßen und durchzuführen versuchen. Elterliche Reaktionen sind dabei nicht unterstützend im Gegenteil: Während die Eltern im besten Fall dem Kind Raum zum eigenständigen Erzählen, Argumentieren oder Erklären zugestehen (vgl. Raumlassen und Akzeptieren in Aufgabenkontexten, Quasthoff \& Kluger in diesem Band), zeigt sich häufig, dass sie kindseitige Beiträge ignorieren und/oder suspendieren und aus der entsprechenden Diskursaktivität hinaussteuern. Letzteres lässt sich außergewöhnlich deutlich im obigen Ausschnitt beobachten: Elterliches Schweigen bzw. verzögerte Reaktionen (Z. 17 bzw. 19, Z. 31, 40, 43), Bekundungen von NichtWissen in der Funktion von Accounts (Z. 19, 44) sowie emphatisches Insistieren (Z. 36) stellen allesamt Mittel dar, mit denen die Eltern sich den kindseitig gesetzten, potenziell argumentativen Zugzwängen entziehen und deren (weitergehende) Bearbeitung zurückweisen. Dies wird insbesondere auch deutlich an zusätzlichen prosodischen Kontextualisierungshinweisen, mit denen Mutter und Vater gesprächsstrukturelles Disalignment zum Ausdruck bringen (z. B. Stöhnen, langsames, gedehntes Sprechen mit mittel-fallender Endtonhöhenbewegung, extra starke Akzente zur Markierung von Dissens) (Morek 2016b). Derlei Gesprächsaktivitäten behandeln die kindseitigen diskursiven Vorstöße als kontextuell unpassend, illegitim und/oder irrelevant und degradieren das Kind zu einem Gesprächspartner, der gerade kein „recognized member“ (Ochs \& Schieffelin 2012: 7) mit potenziell gleichen konversationellen Rechten und Pflichten ist. Entsprechend produzieren Stefans Eltern - ganz anders als etwa 
Alinas Mutter in Beispiel 1 - auch keine gesprächsraumeröffnenden Äußerungen wie etwa ,Wofür bist du?‘ oder ,Warum einen Ball?‘, mit denen argumentative Anforderungen an Stefan gestellt würden. Die begründeten Einwände des Vaters instanziieren allenfalls einseitig elterliche Begründungspflicht (vgl. Heller 2012). Deren - potenzielle - Zugkraft in weitere argumentative Elaborierung hinein wird darüber hinaus durch die Kontextualisierung des Austausches als ,nicht argumentationsorientiert‘ ausgebremst. Das Ausbleiben weiterer Begründungen Stefans nach solchen Elterneinwänden belegt dies auf instruktive Weise.

Nichtsdestotrotz lassen sich einige Beobachtungen dazu machen, welche Mittel und Formen beim Kernjob ,Begründen“ in den Bereichen Vertextung und Markierung genutzt werden. Auf Seiten der Eltern finden wir als semantischpragmatische Mittel Widerlegungen (z. B. durch eine auf den Einzelfall bezogene Vermutung, Z. 33) und Insistieren (Z. 36); daneben Bekundungen von Nicht-Wissen als nicht-argumentatives Mittel. Stefan nutzt eine isolierte Negation als Widerspruch (Z. 34), Betteln (Z. 35), eine Gegenbehauptung (Z. 38), und er führt Beispiele an (Z. 39, 41). Die verwendeten Mittel sind in ihrer semantisch-pragmatischen Funktion für die Argumentation allerdings teils schwer rekonstruierbar, was auch mit dem nahezu vollständigen Fehlen expliziter Markierungen zusammenhängen dürfte. Der jeweilige argumentative Status der einzelnen Äußerungen ergibt sich ausschließlich aus deren sequenzieller Platzierung, wobei der Prosodie eine stützende Funktion zukommt (z. B. Etablierung von Adversativität durch Kontrastakzent, Z. 38). Insgesamt ist die Argumentation sehr enthymematisch, denn es müssen durchgängig Kontext-Implikate mitverstanden werden (Klein 1980, vgl. Heller 2012: 85). Die gemeinsame Vertextung, d.h. der inhaltliche Aufbau dieser Mittel zu einer globalen Argumentation, erscheint hier eher pingpong-artig als Austausch von Behauptungen bzw. Begründungen und Widersprüchen bzw. Widerlegungen. Strukturell komplexe Begründungen oder flankierende Mittel des Erläuterns finden sich ebenso wenig wie konzessive Strukturen oder andere Konvergenzverfahren (wie sie in Beispiel 1 vertreten waren).

Insgesamt aktualisieren also die Beteiligten ein sehr begrenztes Repertoire an semantisch-pragmatischen Mitteln und sprachlichen Formen, von denen einige sogar nicht genuin argumentativ sind, sondern auch im Rahmen anderer Formen der Dissensbearbeitung zum Tragen kommen (z. B. Widersprechen, Insistieren, vgl. Goodwin \& Goodwin 1987; Morek 2014). Sie dürften unterrichtlich kaum oder wie das rein exemplarische Argumentieren (vgl. dazu Spiegel 2003) - nur eingeschränkt zu nutzen sein.

Dass kaum i. e. S. wechselseitig argumentiert wird, wird in beträchtlichem Maße durch die eingeschränkte elterliche Responsivität und Unterstützung bedingt. Trotzdem lässt sich auch am obigen Ausschnitt zeigen, welchen Anteil das Kind daran hat, dass letztlich kein argumentativer Gesprächskontext etabliert wird. Wiederum 
kommt hier der sequenziellen Kontextualisierung und deren Markierung eine Schlüsselrolle zu. Wie eingangs bereits herausgestellt, ist die obige Sequenz uneindeutig kontextualisiert, nämlich zwischen a) dem Bitten um einen Ratschlag, b) dem Stellen einer Erlaubnisfrage und c) dem argumentierenden Aushandeln divergenter Geltungsansprüche. Dafür dürften mehrere Vagheiten und Inkonsistenzen bei der lokalen Einbettung des (argumentativen) Vorstoßes in den laufenden Gesprächskontext ausschlaggebend sein. So knüpft Stefan zwar mit der Frage „was soll ich (dem/ ihm) denn GE::ben;“ (Z. 16) implizit an den bestehenden thematischen Fokus an. Jedoch findet eine Fokus-Verschiebung statt, und zwar von der zeitlichen Planung des anstehenden Wochenendes zur praktischen Vorbereitung des für das Wochenende terminierten Geburtstagsbesuchs. Diese eingeschränkte thematische Kohärenz (Kern \& Quasthoff 2005) markiert Stefan nicht eigens (etwa: ,apropos Wochenende: . . .). Im Gegenteil: Die „rückwärtskonnektive“ Modalpartikel denn signalisiert vielmehr direktes Anschließen an Vorheriges (vgl. Zifonun, Hoffmann \& Strecker 1997: 503). Erschwert wird die inhaltliche Einordnung dieser Frage Stefans auch dadurch, dass die Referenz („dem/ihm“, Z. 16) nicht anaphorisch gedeutet werden kann und somit unklar ist, um wessen Geburtstag es geht. Hinzu kommen sequenziell ungünstige Platzierungen der aktivitätseröffnenden Äußerungen. So produziert Stefan seine Auftaktfragen zunächst zwei Mal in Überlappung zu elterlichen Äußerungen (Z. 15, Z. 24), z. B. als die Eltern noch mit der dyadischen Klärung des genauen Termins des Geburtstags beschäftigt sind. ${ }^{20}$ Dies lässt in Zusammenhang mit der mangelnden thematischen Kohärenz vermuten, dass Stefan den laufenden Gesprächskontext in seinem sukzessiven Aufbau ggf. nicht ausreichend verarbeitet und berücksichtigt, um eine neue Aktivität anzuschließen.

Vor allem aber ergeben sich Inkonsistenzen im Bereich der gattungsspezifischen globalen Projizierung, die v. a. durch die für die dreimalige Eröffnungsfrage gewählte Konstruktion aus Modalverb sollen + Sprecherdeixis 1. Person Singular entstehen (Z. 16, 24, 29). ${ }^{21}$ Diese oberflächlich auf einen Ratschlag zielende Frage eröffnet gerade keinen Raum zum wechselseitigen Argumentieren mit globaler

20 Diese Sequenz (Z. 21-23 sowie 25-28) ist insofern äußerst bemerkenswert, als die Eltern damit den ursprünglichen thematischen Fokus wieder einsetzen, den Stefan zu verschieben versucht hatte. Sie scheinen also den Schwenk weg von der zeitlichen Seite der Terminplanung (noch?) nicht mitvollzogen zu haben.

21 Sollen findet normalerweise in Fällen Verwendung, wo ,jemandem von einer anderen Person [. . . ] ein Handlungsziel auferlegt ist“ (Hoffmann 2013: 295). Anders indes lägen die Dinge bei der Konstruktion Sollen wir . . . ?, mit der standardmäßig Vorschläge etabliert werden. Da Vorschläge auf eine offene Handlungsplanung zielen (Zifonun, Hoffmann \& Strecker 1997: 137), eröffnen sie - anders als eine Bitte um einen Ratschlag - Raum für wechselseitiges Argumentieren. 
Reichweite. Vielmehr setzt sie das einlinige, lokale Beantworten einer Frage relevant und macht allenfalls einseitiges Begründen durch den Adressaten sequenziell erwartbar, wie es im konkreten Fall auch durch den Vater geschieht (Z. 33). Der weitere Interaktionsverlauf aber offenbart, dass es Stefan sodann nicht um elterlichen Rat geht, sondern um die Durchsetzung einer von ihm favorisierten Geschenkoption (vgl. Z. 34-42). Dennoch wird der väterliche Einwand gegen den Vorschlag (Z. 33f.) zunächst nicht zum Anschluss eines eigenen Arguments genutzt. Stattdessen verlegt sich Stefan auf die Aktivität des ,Bettelns‘, d. h. er wählt eine nicht-argumentative Handlung.

Die Beobachtung, dass und wie Stefan wiederholt durch seine Äußerungen die Entscheidungsgewalt an die Eltern überträgt, dokumentiert allerdings, dass er im Bereich der sozialen Kontextualisierung durchaus einen Zuschnitt auf die sprachlich-interaktiven Erwartungen vornimmt, wie sie für Tischgespräche in seiner Familie gelten. Mit der Bitte um Rat wählt er nicht nur ein Verfahren, das den Eltern bevorrechtigten Beteiligungs- und Entscheidungsstatus einräumt (vgl. Z. 19: „wei::ß ich AU_no_ni:::ch“), sondern auch eines, das über eine lokale Frage-Antwort-Sequenz zu bewerkstelligen wäre. Damit entspricht sein Vorstoß zum einen der familialen Dominanz lokal organisierten Kommunizierens (vgl. 5.1). Vor allem aber fügt er sich ein in das familiale Gattungsrepertoire, das eben nicht das wechselseitige Aushandeln von Geltungsansprüchen umfasst, wohl aber zahlreiche einseitig auf elterliche Bevorrechtigungen angelegte Formen (z. B. Vorwurf-Rechtfertigungs-Sequenzen, Ermahnungen, Tadel, Belehrungen). Insofern agiert Stefan im Bereich der sozialen Kontextualisierung also durchaus folgerichtig, wenn er keine Argumentation kontextualisiert. Dass seine Bitte um Rat anschließend kollektiv (um)gedeutet wird als Bitte um Erlaubnis (Z. 34-36), dürfte somit ebenso wenig Zufall sein, sondern vielmehr Reflex des spezifischen Gattungsrepertoires dieser Familie.

Inwiefern insgesamt ggf. auch sprachstrukturelle Defizite auf Seiten Stefans eine Rolle für seine hier gezeigten eingeschränkten Diskursleistungen spielen, kann an dieser Stelle nicht überprüft werden. Auffällig ist aber beispielsweise das Fehlen jeglicher kausaler Konnektoren, die ggf. semantisch-pragmatisch dissonante Verwendung des Modalverbs sollen oder auch die Wahl des unterspezifizierten Lexems geben (statt schenken) in der Äußerung „was soll ich (dem/ihm) denn GE::ben;“ (Z. 16).

Insgesamt zeigt die exemplarische Analyse, dass und wie sowohl die Eltern als auch Stefan mit ihren wechselseitig aufeinander bezogenen Gesprächsbeiträgen dazu beitragen, dass kein Argumentieren instanziiert wird, d.h. keine Sequenz wechselseitigen Austauschens von Positionen und deren Begründungen entsteht. 


\subsection{Peer-Interaktionen in Alinas und Stefans Clique}

Wie in Abschnitt 5.1 bereits grob skizziert, unterscheiden sich auch die Peer-Interaktionen von Alina und Stefan sehr deutlich in ihren sprachlich-kommunikativen Praktiken. Dies betrifft sowohl thematische Präferenzen als auch die aktualisierten Gattungen. In diesen unterschiedlichen Repertoires spiegeln sich gruppenspezifische kommunikative Kulturen wider, die sich auf wiederum höherer Ebene in Bezug setzen lassen zum jeweiligen Selbstverständnis der Gruppe.

Alinas Clique besteht aus drei zehn- bzw. elfjährigen Mädchen, die gemeinsam dieselbe fünfte Gymnasialklasse besuchen. Auch die Freundinnen (Lisa, Justine) stammen aus sozial privilegierten Familien. ${ }^{22}$ Im Rahmen der Fragebogenerhebung haben sich die Mädchen selbst den Namen „Shopping Queens“"23 gegeben; als gemeinsame Freizeitaktivitäten nennen sie zuerst ,Shoppen' und ,Eis essen‘. Dass Konsum und stereotype Weiblichkeit für das Selbstverständnis der Clique zentral sind, geben sie nicht nur in der Gruppendiskussion deutlich zu erkennen, wenn sie offen mit ihrem „Shoppingtrieb“ und ihrer Modebewusstheit kokettieren und sich von als „hässlich“ bezeichneten Mädchen abgrenzen. Es zeigt sich auch in den Inhalten der Gespräche, die über weite Strecken von narrativen Sequenzen verschiedenen Typs gekennzeichnet sind (z. B. Lästererzählungen, kollaboratives Erzählen, Fiktionalisieren), wobei Materielles (z. B. Einkaufen, Medienbesitz, Zimmerausstattung, Urlaube) thematisch eine wichtige Rolle spielt. Die aktualisierten sprachlichen Praktiken sind durchgängig gemeinsamkeitsstiftend und auf die Konsolidierung der Ingroup gerichtet; folglich werden im Rahmen verschiedenster Praktiken immer wieder Abgrenzungen von Anderen vorgenommen (v. a. Eltern, Lehrer, Mitschülerinnen und Mitschüler). Darüber hinaus wird „Unsinn machen“ (Fragebogenantwort) bzw. „Spaß haben“ als wichtige Gemeinsamkeit der Gruppe etabliert und über verschiedene Praktiken aktualisiert (z. B. Witze, Frotzeln, Erzählungen über das Stören des Unterrichts). Eine solche Orientierung am gemeinsamen Spaß (vgl. Deppermann \& Schmidt 2001) dokumentiert sich auch sprachlich-stilistisch, z. B. in der Bevorzugung expressiven, szenisch-performativen Sprechens (vgl. Morek \& Quasthoff 2018), das viel jugendsprachlichexpressive Lexik sowie multimodale Ressourcen verwendet.

Stefans Clique besteht aus fünf Jungen im Alter von zehn bis zwölf Jahren, die gemeinsam die fünfte Klasse einer Hauptschule besuchen. Auch diese Clique

22 Sie verfügen wie Alinas Familie über mehr als 200 Bücher im Haushalt; beide Eltern sind jeweils tätig in gehobenen Positionen (Väter) bzw. freiberuflich oder selbstständig (Mütter). 23 „Shopping Queen“ ist der Titel einer seit 2012 auf dem Fernsehsender VOX ausgestrahlten Doku-Soap, bei der Kandidatinnen mit begrenztem Geld- und Zeitbudget um das Shoppen des von einem Modedesigner zu bewertenden besten Outfits kämpfen. 
ist hinsichtlich der sozialen Herkunftsmilieus ihrer Mitglieder homogen: Alle Jungen stammen aus (eher) sozial benachteiligten Familien. ${ }^{24}$ Sie haben sich im Rahmen der Fragebogenerhebung selbst den Namen „Party Boys“ gegeben und begründen diese Wahl im Gruppeninterview damit, dass sie „so Partystimmung und so [seien]“ und gerne „Faxen“ machten. Als gemeinsame Hobbys geben sie mit „Flur-Rennen“, „Fußball“ und „Tischtennis“ körperlich-sportliche Aktivitäten an. Ihre IngroupKommunikation ist vor allem gekennzeichnet durch eine stark kompetitive, um Angriff und Übertrumpfen bemühte Orientierung sowie eine ausgeprägte körperlich-performative Komponente. Dies offenbart sich auch deutlich im kommunikativen Repertoire der Clique (vgl. Tab. 3 und 5): Sequenzen, in denen die Jungen einander ,dissen', also sich kurze, spielerisch-aggressive Verbalduelle liefern (Deppermann \& Schmidt 2001), machen rund ein Drittel der aufgezeichneten Gruppenkommunikation aus. Weitere $20 \%$ der Zeit werden für kurze, rollenspielartige Performances und körperliche Inszenierungen aufgewendet (z. B. körperliche ,Mutproben“; Inszenieren von Spaßkämpfchen, ,Porno‘-Dialogen und Begrüßungen in die Kamera). Insgesamt sind das Brechen mit Erwartungen, Schlagfertigkeit und Performativität charakteristische Anforderungen der Interaktion in dieser Clique. Thematische Ressourcen sind - neben dem unmittelbar Situativen - körperliche Faktoren (z. B. Muskeln), mediale Erfahrungen (z. B. Facebook, Rapper), sprachlich-stimmliche und körperliche PerformanceFähigkeiten (z. B. rappen können, rülpsen können) sowie das spielerische Beleidigen mit Hilfe ethnischer Kategorisierungen (z. B. als ,Deutschbauer“, ,Kurde“, ,Zigeuner'). Sprachlich-stilistisch sind darüber hinaus v. a. die Verwendung von Ironie, Hyperbolik und ethnolektalem Sprechen (Auer 2003) zu erwähnen.

Für den hier exemplarisch vorzuführenden Vergleich der interaktiven Erfahrungen in den Peergroups wurden zwei Sequenzen ausgewählt, die einer der im DisKo-Projekt fokussierten Gattungen zugeordnet werden können (vgl. Abschnitt 3) und an denen die Fokuskinder Alina und Stefan aktiv verbal partizipieren. Aufgrund der Datenlage fiel die Auswahl damit auf zwei Ausschnitte, in denen es um die Bearbeitung einer (im zweiten Fall: vermeintlichen) Wissensasymmetrie geht, die also (vermeintlich) explanativ sind. Damit wird für die Cliquen eine Praktik fokussiert, die zwar nur selten (Alina) bzw. gar nicht (Stefan) für die Peer-Gespräche kodiert werden konnte, aber für die Frage nach schulrelevanten Diskurserwerbser-

24 Die Haushalte der Freunde verfügen laut Fragebogenerhebung ebenso wie Stefans Familie über vergleichsweise wenige Bücher (11-25 Bücher bzw. 26-100). Die Eltern sind entweder Hausfrau oder arbeiten in Bereichen wie Reinigungsservice, Montage/Bau, Sicherheitsdienst, herstellende Fabriken und Gebrauchtwagenhandel. Zwei Jungen (Emre und Ahmet) sprechen in ihren Familien z. T. auch eine andere Sprache als Deutsch. 
fahrungen besonders relevant ist, weil sie in Unterrichtsinteraktionen die häufigste Diskursaktivität darstellt (Heller et al. 2017; Morek 2016a).

Die hier zu präsentierende Analyse der beiden Ausschnitte fokussiert analog zu den Familieninteraktionen - die Fragen, wie gesprächsstrukturelle Aufgaben bearbeitet werden, welche Interaktionsmuster dabei zutage treten und wie das Kontextualisieren, Vertexten und Markieren vollzogen wird; dabei wird wiederum nach den Anteilen der Fokuskinder an der Hervorbringung des spezifischen sequenziellen Kontextes gefragt.

\subsubsection{Alinas Peer-Interaktionen: Erklären als Spagat zwischen Asymmetrie und Gruppenkonsolidierung}

Der folgende Transkriptausschnitt setzt ein, als Alina dabei ist, von einem Urlaub zu erzählen. Dabei entwirft sie sich selbst als Protagonistin, die am Pool relaxt (Z. 46), außergewöhnliche Getränke konsumiert (Z. 44-45, 48) und Wellness-Behandlungen genießt (Z. 47). Sie aktualisiert also exakt die oben beschriebenen Relevanzen der „Shopping Queens“, und zwar auch formal (z. B. ey; ich SCHWÖR; so: geil; voll HEISS da; körperliches Vormachen des Trinkens). Als sie sich selbst als Konsumierende positioniert und von ihrem als Aktivsportler positionierten Vater abgrenzt (Z. 66), kommt es zu einer Erklärung des Spiels „Glow Hockey“, ${ }^{25}$ und zwar in Form einer eingebetteten Nebensequenz innerhalb der Urlaubserzählung:

(3) Glow-Hockey (25w-C6w-Gy2), Al: Alina (11 J.), Ju: Justine (11 J.), Li: Lisa (10 J.)

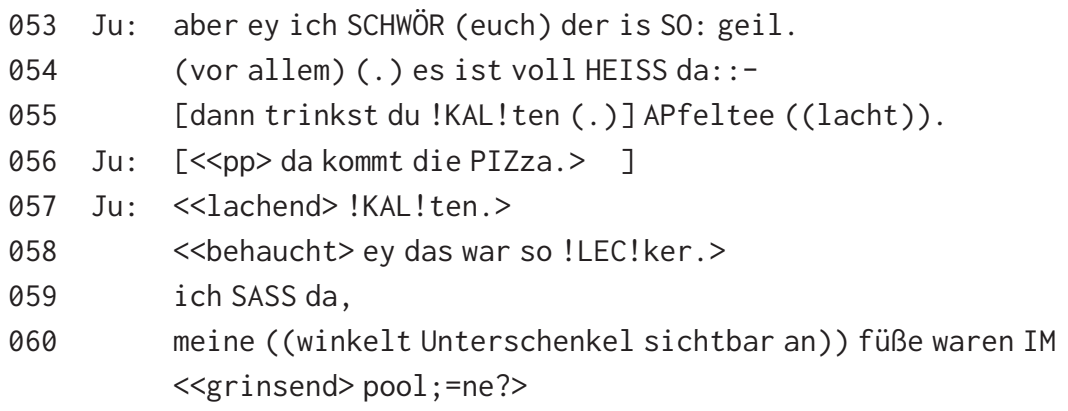

25 Bei „Glow Hockey“ handelt es sich um sog. Air-Hockey, ein vornehmlich in Spielhallen zu findendes Spiel für zwei Spielende, bei dem ein Puck auf einem Tisch mit Hilfe spezieller Rundschläger ins gegnerische Tor befördert werden muss. Der Puck gleitet dabei durch einen Luftkisseneffekt auf der mit Löchern perforierten Tischoberfläche. Glow Hockey ist der Name einer Air-Hockey-App für Smartphones. 
061 die äh wollten (. ) erst GLEICH massieren.

062 ((imitiert zwei Mal Trinkgeräusch und führt imaginären Becher zum Mund))

063 ((drittes Trinkgeräusch und Trinkbewegung, nun übertrieben))

$064 \mathrm{Ju}$ : [hehehe]

065 Li: [hehehe]

$066 \mathrm{Al}$ : und mein vater hat <<monoton, $t>$ irgendwas geSPIELT; >

067 irgendwie so GLOW hockey <<grinst> oder wie das heißt.> |

((angedeutete, schiebend-hämmernde Bewegung mit rechter Hand))

$068 \quad(--)$

$069 \mathrm{Li}$ : ((m. gerunzelter Stirn + verzog. Mund Drehung zu Ju))

$070 \mathrm{Ju}: \quad<$ halb kopfschüttelnd> aHA.>

$071 \mathrm{Al}$ : dieses TISCHhockey da.

((stärkere schiebend-hämmernde Bewegung mit rechter Hand))

072 Li: <<halb kopfschüttelnd> JA: : >

073 [wir ver ]stehn dich !GANZ! GU: :T.>

$074 \mathrm{Ju}:$ [ach so JA]

$075 \mathrm{Al}:$ ((lacht))

$076 \mathrm{Ju}$ : ach DIEses (.) dieses wo man [wo man mit diesen-]

((Ausholbewegung mit rechter Hand))

$077 \mathrm{Al}$ :

[ja: : wo_e ]

$078 \mathrm{Al}$ : GELD reinwerfen muss, =

079 =und dann diese TEIle da [weg. ]

((schiebend-hämmernde Bewegung mit rechter Hand))

$080 \mathrm{Ju}: \quad$ [ach SO; ]

081 Ju: <<kopfschüttelnd, bedauernd> NEE das kenn ich nicht.>

$082 \quad(0.9)$

$083 \mathrm{Ju}: \quad(($ kichert $))$

$084 \mathrm{Al}$ : ((grinst)) DA: : : is_n TISCH;

((Arme zu weitem Rechteck aufgespannt))

085 da is sowas wie n!PUCK!?

((zeigt Dicke aus Zeigefinger und Daumen geformt)) 
086

und ZWEI teile zum SCHIEben;>

1

((zwei erhobene Fäuste in Schiebebewegung))

087 und dann muss man das ins gegnerische [TOR bringen.]

((schiebend-hämmernde Bewegung mit rechter Hand))

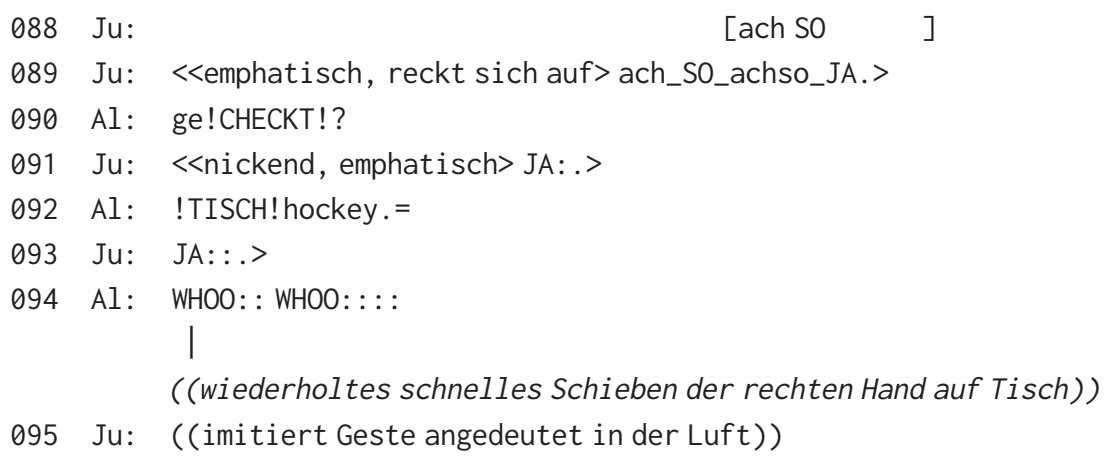

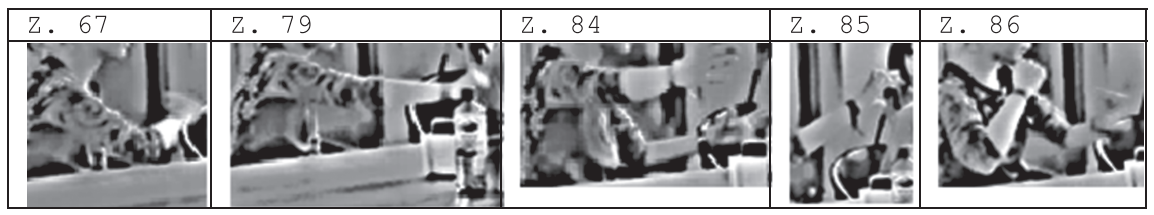

Abb. 1: Screenshots ausgewählter Gesten (vgl. Transkript 3) ${ }^{26}$.

Bei der Sequenz ab Z. 67 handelt es sich um eine Spielerklärung, d. h. um ein Erklären-WAS (Klein 2009). Dabei werden die für konversationelles Erklären konstitutiven Gesprächsaufgaben (Morek 2012) interaktiv von den Mädchen bearbeitet: ,Inhaltliche Relevantsetzung‘ (Z. 66), ,Konstituieren eines Explanandums‘ (Z. 67-81), ,Erklären` (Z. 84-87), ,Abschließen des Erklärens` (Z. 8895). Dabei zeigt sich auf Seiten Alinas - wie schon in der Familie - eine hohe Eigenleistung, die aber wiederum flankiert wird von unterstützenden Zuhöraktivitäten ihrer Mitinteraktantinnen.

Den Erklärgegenstand Glow Hockey setzt Alina im Rahmen ihrer Erzählung selbst relevant (,irgendwie so GLOW hockey «grinsend > oder wie das heißt. >“, Z. 67). Ihre begleitende ikonische Geste (,angedeutete schiebend-hämmernde

26 Die Peer-Interaktionen wurden, anders als die Familieninteraktionen, nicht nur audio-, sondern auch videographiert; auszugsweise werden in den entsprechenden Transkripten dieses Beitrags gestische Mittel durch Standbilder repräsentiert, um den szenischen Charakter der Gesprächs- bzw. Erklärsequenzen zu illustrieren. 
Bewegung mit rechter Hand“) und der anschließend gelassene Slot (Z. 68) lassen darauf schließen, dass sie etwaiges Nicht-Verstehen auf Seiten ihrer Zuhörerinnen ggf. bereits antizipiert. Daraufhin treten die drei Mädchen in eine längere Klärungssequenz ein, in der sie sich zunächst darüber verständigen, ob das potenzielle Explanandum ein tatsächliches ist (Z. 69-81). Das interaktive Aushandeln von Erklärbedarf erstreckt sich also über mehrere Züge, wobei Lisa und Justine als prospektive Erkläradressatinnen aktiven Anteil an der Bearbeitung dieser Gesprächsaufgabe haben: Zunächst zeigen sie mimisch (Z. 69) und durch verständnisbezogene verbale Rückmeldungen Irritation an, und zwar auf scherzhaft-ironisierende Weise (,aHA.“, Z. 70, „wir verstehn dich !GANZ! GU::: T.“, Z. 73); anschließend setzt Justine zu einer referenzbezogenen Verstehensdemonstration an (,ach DIESES (.) dieses wo man mit diesen“, Z. 76). In Reaktion auf diese Zuhöraktivitäten liefert Alina bereits in dieser Klärungsphase einzelne explanative Äußerungen: So ersetzt sie den ursprünglich erwähnten Eigennamen durch eine übergeordnete Gattungsbezeichnung („dieses TISCHhockey da.“, Z. 71), illustriert erneut durch eine ikonische Geste (ebd. und Z. 79) und expliziert die Grundhandlungen der Inbetriebnahme sowie des Spiels als solchem („wo_e GELD reinwerfen muss, = und dann diese TEIle da weg. “, Z. 77-79). ${ }^{27}$

An dieser Stelle nun manifestiert sich für die Mädchen endgültig Erklärbedarf: Justine markiert das Nicht-Zutreffen ihrer Vermutung (,ach SO“, Z. 80) und legt ein Wissensdefizit offen („NEE das kenn ich nicht.“, Z. 81). Damit setzt sie einen impliziten globalen Zugzwang an Alina, nun eine Erklärung des in Frage stehenden Spiels anzuschließen, und nimmt selbst die Gesprächsrolle der primären Erkläradressatin ein. Diesem Zugzwang kommt Alina mit der Produktion eines explanativen Äußerungspaketes nach (Z. 84-87). Ihre Erklärung besteht aus dem Nennen des Spielmaterials (vgl. Stude 2003) - „TISCH“, „sowas wie n !PUCK!“ und „ZWEI teile zum SCHIEben“ (Z. 84-86) - sowie des Spielgedankens („muss man das ins gegnerische TOR bringen.“, Z. 87). Viele ikonische Gesten illustrieren dabei das Gesagte (vgl. Abb. 1). Während des Erklärens blicken die Freundinnen Alina an und hören ihr zu. Im Abschluss bringt sich Justine als primäre Adressatin dann wieder verbal ein. Emphatisch signalisiert sie Verständnis (,ach_SO_ach_so_JA.“, Z. 89) und tritt mit der Erklärerin in eine Rückkopplungsschleife ein. Die beiden vergewissern sich sowohl verbal (Z. 90-93) als auch gestisch (Z. 94-95) des kommunikativen Erfolgs der Erklärung und beenden diese gemeinschaftlich.

27 Implizit wird damit das Spiel auch kategorisiert, nämlich als ,Münzspielautomat‘ und Spiel mit Schlägern. Unklar bleibt allerdings an dieser Stelle, ob es sich um ein Torspiel oder Rückschlagspiel handelt; diese Information wird erst später geliefert (Z. 87). 
Auf Ebene der gesprächsstrukturellen Jobs zeigt sich also, dass Alina sehr eigenständig agiert, insofern sie die Erklärung selbst vorbereitet, durchführt und deren Abschluss steuert. Allerdings erfährt sie interaktive Mitarbeit durch ihre Peers, und zwar v. a. beim Konstituieren des Explanandums, das sozusagen den neuralgischen Punkt für den Eintritt in Erklärungen ausmacht: Zum einen ratifizieren Lisa und Justine durch ihre Zuhöraktivitäten das Explanandum und setzen globale Zugzwänge zum Erklären. Zum anderen unterstützt Justine sogar aus der Zuhörerinnenrolle heraus die Erklärerin. So liefert Justines auf Verstehensdokumentation angelegte Zuhöreräußerung „dieses wo man mit diesen“ (Z. 76) nicht nur einen Ansatzpunkt für eine „operationale Erklärung“ (Quasthoff \& Hartmann 1982: 104), sondern modelliert auch deren sprachlich-formale Umsetzung mittels generischem man. Mikrogenetisch zeigt sich, dass die Erklärerin, Alina, in Reaktion darauf tatsächlich Spielhandlungen in verallgemeinerndem Format vertextet (Z. 77-79). Somit lässt sich hier - in Analogie zu den für Erwachsenen-Kind-Interaktionen beschriebenen Interaktionsmustern - durchaus von einem Fordern und Unterstützen auch durch die Peers sprechen. Allerdings gestaltet sich die Art der fordernden und unterstützenden Gesprächsaktivitäten äußerst subtil und dürfte ggf. sogar peergruppenspezifisch sein: Beispielsweise finden sich implizite und sogar ironische Mittel des Konstituierens von Erklärbedarf anstelle erklärungsorientierter Ergänzungsfragen (z. B. ,Was ist Glow Hockey?`) oder gar Explizierungen von Zugzwängen (,Erklär mal, was das ist!'). Dennoch werden hier durch die Peers explanative Anforderungen etabliert und wird interaktive Unterstützung bei deren Bewerkstelligung geleistet.

Betrachtet man für die von Alina in der Clique gelieferte Erklärung die Vertextungs- und Markierungsebene, zeigen sich zahlreiche gattungsspezifische Realisierungen: Inhaltlich ist die Erklärung hierarchisch aufgebaut, insofern zunächst mit „Tischhockey“ ein Genus proximum (Quasthoff \& Hartmann 1982) angeführt wird, eine übergeordnete Gattungsbezeichnung (Z. 71). Darauf folgt eine operational angelegte Explizierung zentraler Spiel(inbetriebnahme)handlungen (Z. 77-79). Diese wird mit Übernahme der primären Sprecherinnenrolle ergänzt um eine Auflistung der materiellen Bestandteile des Spiels (Z. 84-86), wobei die zentrale Spielhandlung erneut Erwähnung findet („zum Schieben“, Z. 86). Abschließend wird der Spielgedanke genannt (,ins gegnerische TOR bringen.“, Z. 87). Es lässt sich also eine Bewegung nachzeichnen, die von der kategorisierenden Verortung bzw. vom Anknüpfen an Bekanntes (,Tischhockey') über die Entfaltung einzelner wesentlicher Merkmale hin zur Ziel- bzw. Funktionsbestimmung verläuft. Die akzentuierte Wiederholung des Explanandums im Abschluss („!TISCH!hockey.“, Z. 92) markiert i. S. einer schließenden Klammer das Ende der Erklärung.

Wenngleich also die vorliegende Erklärung zunächst inkrementell und interaktiv vertextet wird und insgesamt relativ stark kondensiert wird, so entspricht sie deutlich der gattungsspezifischen und bereits nicht mehr bloß linear-reihen- 
den Vertextung von Spielerklärungen (Stude 2003). Für die Markierungsebene zeigt sich ebenfalls, dass Alina erklärtypische sprachliche Formen verwendet. Dazu zählen z. B. das Modalverb müssen (Z. 78, 87) und das generische Pronomen man (Z. 87), die zur Explizierung von Spielhandlungen genutzt werden, zudem die Vergleichsprozedur so was wie (Z. 85) sowie die Präpositionalphrase zum Schieben als Ausdruck einer Funktionsbestimmung (Rehbein 1984: 88). Insgesamt wählt Alina einen eher demonstrierenden Stil (Kern 2003), der sich stark auf deskriptiv-illustrative Begleitgestik stützt. Er dürfte der peergroupspezifischen Präferenz für performativ-szenisches Sprechen ebenso entgegenkommen wie der Gebrauch sprachlicher Formen, die Informalität und Jugendsprachlichkeit indizieren, wie etwa das generisch verwendete $d u$ in phonologisch geschwächter Variante (Z. 77), das Passepartout-Wort Teile (Z. 79), das Lexem checken (Z. 90) und der onomatopoetische Ausruf WHOOO::: WHOOO:::: (Z. 94). Gerade im Abschließen der Erklärung wird so eine Informalisierung und fast übertriebene Expressivität bei der wechselseitigen Verständnissicherung ablesbar.

Nicht nur in diesem Einschluss szenisch-expressiver Formen, sondern auch in der hinführenden sequenziellen Kontextualisierung des Erklärens offenbart sich ein spezifischer Zuschnitt des Erklärens auf die kommunikativen Anforderungen dieser Clique. Die Erklärung ist eingebettet in eine Urlaubserzählung, die der Konsolidierung der auf Konsum und Weiblichkeit gerichteten Gruppenidentität der Shopping Queens dient. Lanciert wird die Gattung des Erklärens in diesem Zusammenhang auf eine Weise, die die Ingroup-Kohärenz stärkt und (epistemische und kommunikative) Unterschiede reduziert: Zum einen ergänzt Alina die thematische Fokussierung eines (potenziellen) Explanandums durch Kontextualisierungshinweise: Mit Heckenausdrücken (,irgendwie so“, „oder wie das heißt“, Z. 67) und grinsendem Sprechen etabliert sie ,Glow Hockey“ als etwas - auch für sie selbst - Distantes. Zum anderen liefert sie einen gestischen Hinweis, der auf die beiläufige Kompensation etwaigen Nicht-Verstehens gerichtet ist. Insgesamt wird ,Glow Hockey somit eingeführt als etwas, was man als Mitglied dieser Gruppe durchaus nicht kennen mag. Zudem bezieht Alina die Freundinnen gleich zu Beginn interaktiv mit ein, indem sie nach der thematischen Fokussierung einen Slot für Zuhörerreaktionen lässt (Z. 68) und nach Justines Display von Nicht-Wissen (Z. 81) kurz wartet, ehe sie mit der Produktion eines explanativen Äußerungspaketes beginnt (Z. 84).

Insgesamt lässt sich also auf Seiten Alinas Zurückhaltung beim Einstieg in das konversationelle Erklären beobachten. Es werden zunächst - inkrementell und in Zuschnitt auf die verbalen und nonverbalen Reaktionen der Mitinteraktantinnen einzelne, explanativ orientierte Informationen angeboten (Z. 71, 77-79 sowie die Geste in Z. 67 und 71), statt z. B. mit einer Ankündigung wie „passt auf!“ oder „also, das ist . ..“ den Anschluss einer global strukturierten Erklärung direkt zu projizieren (vgl. dazu Quasthoff 1985). Bedient wird der implizite globale Zug- 
zwang von ihr erst, als sich tatsächliches Nicht-Wissen auf Seiten der Peers manifestiert („NEE das kenn ich nicht.“, Z. 81), wobei selbst dann die einleitende Markierung des explanativen Äußerungspakets subtil ausfällt (lediglich durch Akzentuierung und Längung des eröffnenden Lokaldeiktikums „DA:::“, Z. 84). Eine solche Vermeidung allzu starker Gattungsprojektion ermöglicht es, ein etwaiges epistemisches Gefälle (Keppler \& Luckmann 1991) zwischen sich und den Freundinnen zu nivellieren und so gruppeninterne Hierarchien zu minimieren. Anhaltende Aufmerksamkeit sowie aktives Zuhören auf Seiten der Freundinnen dokumentieren dabei den interaktiven Erfolg von Alinas Erklärung.

\subsubsection{Stefans Peer-Interaktionen: Ironisieren und Distanzieren vom Erklären}

Der aus Stefans Peer-Interaktionen ausgewählte Ausschnitt beginnt mit einer für die Party Boys typischen Diss-Sequenz: Bruno wird von Ahmet und Florian in spielerisch-ironischem Modus rassistisch beleidigt, und zwar als „Zigeuner“ bzw. „halb Zigeuner“ (Z. 7, 10). Nachdem er den Angriff pariert ((,,junge du has_n) ziGEUner alter.“, Z. 12), kommt es zu einer Nachfrage, die ein ErklärenWAS konditionell relevant zu machen scheint (Z. 13).

(4) Zigeuner (09 m-C3m-H1), Br: Bruno (10 J.), Ah: Ahmet (11 J.),

Fl: Florian (12 J.), Mi: Milan (12 J.), St: Stefan (11 J.)

$006 \mathrm{Br}:=$ ich bin halt Pole.

007 Ah: und ziGEUner.

$008 \mathrm{Fl}:$ [hä:: :? ]

$009 \quad[(($ kollektives Kichern $))]$

$010 \mathrm{Fl}$ : ich dacht du wärst ha äh halb ziGEUner.

011 ((kollektives Kichern))

$012 \mathrm{Br}$ : (junge du has_n) ziGEUner alter.

$013 \mathrm{Mi}$ : <<an Fl> was IST eigentlich zigeuner;>

$014 \mathrm{Fl}$ : ((schnalzt)) stell dir VOR-=

015 ey_sch bin <<Rappergeste, kauend $>$ ) $>$ n DÖner.

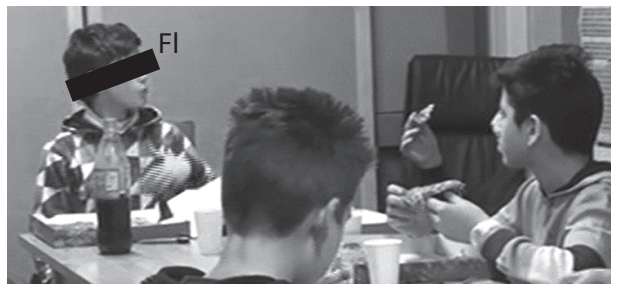




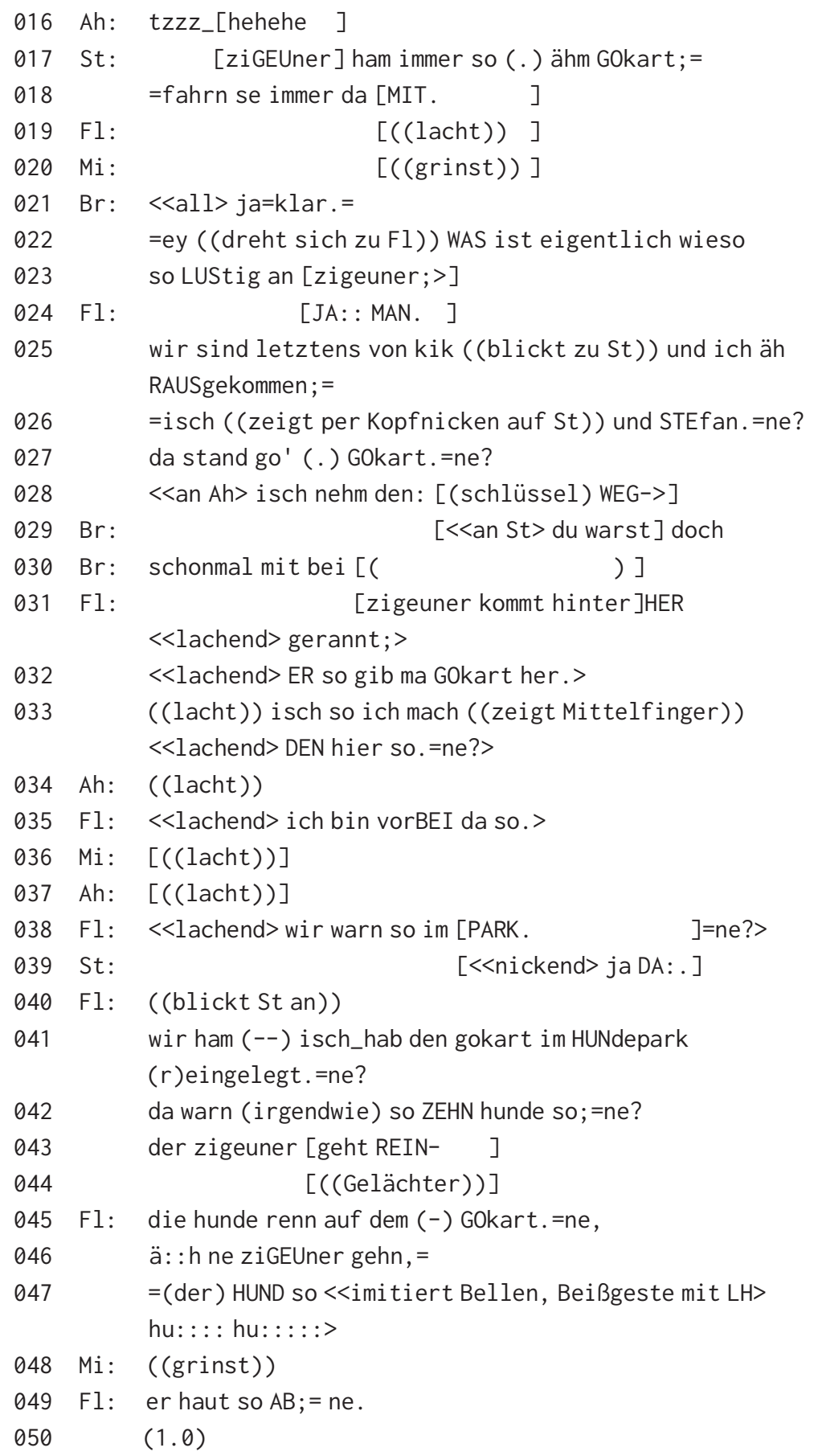


$\mathrm{Br}$ : ja das war das war_n ruMÄnischer zigeuner.=

052 =die sind HERBST scheiße.=

Weil Stefan hier - wie in der gesamten Cliquen-Interaktion - kaum aktiv verbal partizipiert, kann die nun folgende Analyse nicht in gleicher Weise, wie dies bei den bisherigen Beispielen geschehen ist, auf ihn als Fokuskind fokussieren. Es geht daher im Folgenden primär darum, aufzuzeigen, inwiefern Sequenzen wie die obige keine Erwerbskontexte für schulisch anschlussfähige Erklär- und Diskurspraktiken darstellen. Den Rahmen der Analyse bilden auch hier die unter 5.3.1 aufgeführten Jobs des Erklärens.

Inhaltlich relevantgesetzt wird das Thema ,Zigeuner‘ während der von Ahmet und Bruno vollzogenen Diss-Aktivität. Milans Frage „was IST eigentlich zigeuner;“ (Z. 13) ähnelt nun scheinbar einer typischen Frage nach einer Bedeutungserklärung (Morek 2012). Dass hier jedoch gerade keine ernsthaft auf die Behebung eines Wissens- bzw. Verstehensdefizits angelegte Frage vorliegt, wird sowohl durch deren sequenzielle Position als auch das Turn-Design nahegelegt: Milan hat der Diss-Sequenz bis dato nur als unbeteiligter Beobachter beigewohnt. Seine Äußerung nimmt nun eine Verschiebung des bisherigen gesprächskontextuellen Fokus zugunsten eines neuen Fokus vor, wie die Akzentuierung der Kopula IST sowie die Verwendung der fokussierenden Modalpartikel eigentlich signalisieren. Seine bilateral an einen der Angreifer gerichtete, auf die metasprachliche Ebene wechselnde Frage setzt diesen unter einen thematisch und kommunikativ defokussierenden Zugzwang. Dadurch hat sie das Potenzial, die Fortsetzung des verbalen Beleidigens von Bruno zu unterbinden. Ähnliche Fälle von meta-commentary in rituellen Beleidigungssequenzen hat auch Goodwin (2006: 10) beobachten können (z. B. „Is that your favourite word or something?“" nach der Beleidigung ,whore‘). ${ }^{28}$

Und tatsächlich wird der entsprechende Zugzwang von Florian nicht im Sinne einer Bedeutungserklärung in ernsthafter Modalität bearbeitet (Z. 14f.). Zwar projiziert er mit „stell dir VOR“ auf formelhafte Weise den Beginn einer deskriptiven Vertextung, dann jedoch folgt eine stilisierte, ironisierende Äußerung, mit der offenbar stereotype Verhaltensweisen der in Frage stehenden ethnischen Gruppierung im Sinne eines Embodiment vorgeführt wird (,ey_sch bin «Rappergeste, kauend > () >n DÖner. “, Z. 15). Die Stilisierung wird para- und nonverbal geleistet

28 Dass hier nicht ernsthaft nach der Wortbedeutung von ,Zigeuner' gefragt wird, wird außerdem noch durch die Tatsache gestützt, dass in den Aufnahmen der „Party Boys“ das Wort ,Zigeuner in mehreren anderen Diss-Aktivitäten rege Verwendung findet. Zudem wird der in der Frage „Was ist eigentlich Zigeuner?“ enthaltene Zugzwang vom Diss-Opfer Bruno später deutlich dissensorientiert re-instanziiert („WAS is eigentlich wieso so LUStig an zigeuner.“, Z. 22f.). 
durch ostentatives Kauen (und daraus resultierende unverständliche Aussprache), die begleitende Coolness-indizierende ,Rappergeste‘ sowie stilistisch-verbal durch ethnolektales Sprechen („ey_sch bin“). Das Stereotyp der Unkultiviertheit wird damit nicht nur inhaltlich durch die Referenz auf das Verzehren einfachen Fastfoods geleistet, sondern auch körperlich inszeniert. Insofern liefert Florian keine eigentlich auf eine Behebung einer epistemischen Asymmetrie gerichtete Erklärung, sondern eine auf die cliquentypischen Zwecke der Unterhaltung und der Gruppenkonsolidierung qua Alteritätsabgrenzung zugeschnittene Performance. Dass es ihm dadurch gelingt, den von Milan im Rahmen der Diss-Sequenz platzierten Zugzwang auf interaktiv erfolgreiche Weise $\mathrm{zu}$ deuten und $\mathrm{zu}$ bedienen, wird in der sowohl scherzhaft-empörenden als auch goutierenden Reaktion eines anderen Gruppenmitglieds sichtbar (,tzzzz_ hehehe“, Z. 16).

An dieser Stelle nun tritt erstmals in dieser Gesprächsphase Stefan verbal in Erscheinung. Er kommt auf das vermeintliche Explanandum zurück und führt ,Gokart-Fahren' als (weiteres) wesentliches Merkmal von Zigeunern ins Feld („,ziGEUner ham immer so (.) ähm GOkart; = fahrn se immer da MIT.“, Z. 17 f.). Eine etwaige Ironie seiner Äußerung operiert dabei allein auf dem Weltwissen der Beteiligten (Gokartfahren ist gemeinhin kein definierendes Merkmal der Sinti und Roma), ist entsprechend sprachlich nicht eigens markiert, etwa durch para- oder nonverbale Verfahren. Dass Stefan mit seiner Äußerung auf ein von Florian und ihm geteiltes, singuläres Ereignis anspielt, zeigen Florians anerkennendes Lachen sowie die kurz darauffolgende konversationelle Erzählung eines entsprechenden Vorfalls (Z. 25-49). Diese von den Jungen vorgenommene Ironisierung potentiellen Erklärens spiegelt sich auch in Brunos Reaktion, der ihnen ironisch zustimmt und einen neuen, auf Rechtfertigung angelegten Zugzwang etabliert (,,ja = klar. = ey ((dreht sich zu Fl)) WAS ist eigentlich wieso so LUStig an zigeuner; >“, Z. 22f.). Daraufhin schließt Florian eine lange und offenbar fiktive Belegerzählung an (Z. 25-49), die - wiederum auf ironisierende Weise - die Stereotypisierung und Abwertung dieser Ethnie zu untermauern sucht. Aus Raumgründen kann an dieser Stelle nicht näher auf die inhaltliche und formale Gestaltung dieser konversationellen Erzählung eingegangen werden. Retrospektiv legt sie in jedem Fall offen, dass zu keinem Zeitpunkt ernsthaftes Erklären im Sinne eines kooperativen Behebens von Wissensasymmetrien etabliert war, sondern das wechselseitige Herausfordern, Sich-selbst-Überhöhen und Übertreiben. Das Mittel, das in anderen Kontexten eine Erklärungssequenz eröffnet, dient hier der thematischen Neufokussierung.

Die Interaktionsmuster, wie sie ursprünglich für verständigungsorientierte Erwachsenen-Kind-Interaktionen rekonstruiert wurden, lassen sich offensichtlich nicht ohne Weiteres auf derartige Peer-Interaktionen übertragen, die als soziale Veranstaltung durch das kompetitive, unernste Austragen ritueller Konflikte ge- 
prägt sind. Das spielerische Gegeneinander scheint das Auftreten unterstützender Gesprächsaktivitäten im Grunde zu blockieren: Ein Diss-Adressat kann dem Gegenüber wohl kaum dabei helfen, einen angemessenen ,Diss' ${ }^{`} \mathrm{u}$ liefern. Allenfalls ein ,Fordern‘ lässt sich im obigen Ausschnitt beobachten, und zwar in Form von Milans und Brunos Nachfragen im Rahmen der Diss-Sequenz. Als auf Angriff und Verteidigung angelegte Äußerungen beim Dissen setzen sie allerdings i.d.R. nur lokale Zugzwänge (vgl. Z. 14-15) und zielen auf möglichst schlagfertige Repliken (Deppermann \& Schmidt 2001). Das Anbringen globaler Diskurseinheiten wird dadurch weder eröffnet noch unterstützt. ${ }^{29}$

Insgesamt bieten Sequenzen wie die obige keinen Erwerbskontext für unterrichtlich anschlussfähige, z. B. explanative Diskurspraktiken. Die Analyse hat zutage gefördert, dass in solchen Sequenzen, wie sie sich im Korpus in den benachteiligten Cliquen finden, gerade nicht mit Zugzwängen zum Erklären hantiert wird, die der kooperativen Behebung einer epistemischen Asymmetrie dienten. Vielmehr werden vermeintlich erklär- oder begründungsorientierte Zugzwänge im Rahmen verbaler Beleidigungs- und Schlagfertigkeitsduelle funktionalisiert und spielerisch-unernst (um)gedeutet. Dabei geht es dann gerade nicht um das Explizieren allgemeiner Wissensbestände zum Zwecke des Wissenstransfers, sondern um die Demonstration von Witz und Schlagfertigkeit im Rahmen der gruppeninternen Statusaushandlung. In der Folge werden keine global strukturierten, den Gattungsnormen von Erklärungen entsprechenden Äußerungen produziert. Stattdessen finden sich stilisierende und ironisierende, i.d.R. schnell getaktete lokale Verfahren des Umgangs mit den entsprechenden Zugzwängen. Beispielsweise werden im Rahmen uneigentlichen Erklärens evaluativ gefärbte, performative und ironische Stereotypisierungen angeführt, die gerade kein intersubjektiv gültiges Wissen repräsentieren. Auch im Bereich der sprachlichen Formen werden - abgesehen von Florians Diskursmarker stell dir vor (Z. 14) - keine global- bzw. gattungsrelevanten Ausdrücke für das Erklären verwendet.

29 Das Anbringen etwaiger entsprechender Diskurseinheiten verlangt eigenständiges Kontextualisieren. Während Florian dies offensichtlich zu leisten in der Lage ist (vgl. seine Erzählung in Rechtfertigungsfunktion), gelingt es dem Fokuskind Stefan nicht, lokale Anschlussstellen im Sinne globaler Diskursoptionen für sich zu nutzen. Selbst als Ko-Erzähler tritt er nicht in Erscheinung - und das, obwohl er vom primären Erzähler namentlich als Mitwisser angeführt wird (,isch und STEfan._ne?“, Z.26) und mehrmals angeschaut wird (Z. 25, 26, 40). 


\subsection{Zusammenfassung: Alinas vs. Stefans außerschulische Diskurspraktiken}

Was erbringen nun die hier vorgestellten exemplarischen Analysen von Sequenzen aus den Familien- und Peer-Interaktionen der beiden Kinder mit Blick auf die Fragen 1a-c und 2?

Für Alinas Familieninteraktionen zeigt sich, dass dort eine vielfältige, anregungsreiche sowie unterrichtlich anschlussfähige kommunikative Erfahrungswelt mit interaktiver elterlicher Unterstützung auf gut ausgebaute diskursive Kompetenzen des Kindes v. a. auch im Bereich der Kontextualisierung stößt. Die hohe kindliche Kompetenz trägt ihrerseits zur (Re)Produktion entsprechender diskursiver Interaktions- und mithin Erwerbskontexte bei. Insgesamt weist dabei schon die konsensuelle, abwägende Anlage des Argumentierens eine besondere Nähe zu unterrichtlichem Argumentieren auf. Das fordernd-unterstützende Interaktionsmuster eröffnet Gesprächs- und Übungsräume für Diskursaktivitäten und v. a. für deren strukturellen Ausbau zu komplexer Vertextung, wobei genuin argumentationsspezifische Mittel und Formen zum Tragen kommen. So wird beispielsweise v. a. an der Güte der Argumente gearbeitet und an der Integration möglicher Einwände zugunsten eines mehrperspektivischen Argumentierens. Sprachliche Explizierungen logischer Verknüpfungen und der Gebrauch verschiedener Modalisierungen sind dabei typische bildungssprachliche Charakteristika auf Formenebene (Feilke 2012).

Ihre offenbar familial angeeigneten diskursiven Kompetenzen kann Alina, so zeigt der Blick in ihre Clique, auch in Peer-Interaktionen mit interaktivem Erfolg einbringen. Auch dort lässt sich zeigen, wie interne Ressourcen in Form gut ausgebauter Kompetenzen in den Bereichen (explanativer) Kontextualisierung, Vertextung und Markierung auf diskursiv anregende und unterstützende externe Ressourcen stoßen und dazu führen, im Rahmen informeller Cliquengespräche Gesprächsraum für erklärende Sequenzen zu erlangen. Subtile, $d$. h. teils ironische und implizite Gesprächsaktivitäten der Peers bilden dabei ein peergruppenspezifisches Pendant zu dem für Erwachsenen-Kind-Interaktionen herauspräparierten Interaktionsmuster Forderm und Unterstützen. Bemerkenswert ist, dass es auf diese Weise trotz der grundsätzlich eingeschränkten Kompatibilität mit typischen Orientierungen des doing peer-group zu strukturell komplexen Äußerungspaketen mit dem Ziel des Wissenstransfers unter den Peers kommt. Dabei werden wiederum semantisch-pragmatische Mittel und Formen gewählt, die erklärtypisch sind und eine entsprechend gattungsspezifische Vertextung und Markierung erkennen lassen (z. B. Anknüpfung an Bekanntes, Kategorisierung, deskriptive Entfaltung, Explikation von Regelhaftigkeiten). Diese werden mit körperlich-performativen und jugendsprachlich-expressiven Formen kombiniert und sequenziell so in das übergeordnete Erzählen eingebettet, dass peergroupspezifi- 
sche sprachlich-stilistische Präferenzen in das Erklären inkorporiert werden und an die Kommunikationskultur der Clique angeschlossen wird. Auf diese Weise trägt insbesondere Alinas Kontextualisierungskompetenz dazu bei, das breite Gattungsrepertoire ihrer „Shopping Queens“, das in beträchtlichem Maße v.a. narrative, aber auch explanative und argumentative Diskursaktivitäten umfasst, entscheidend mit hervorzubringen. Somit lässt sich gerade aus der Gegenüberstellung der diskursiven Partizipation ein- und desselben Kindes in unterschiedlichen sozialen Kontexten nachzeichnen, wie interne und externe Ressourcen bei der Hervorbringung sequenzieller Kontexte für Diskurserwerb zusammenwirken.

Entsprechendes zeigt sich - in umgekehrter Wirkweise - auch für den komplementär ausgewählten Fall des Fokuskindes Stefan. Die hier vorgestellte exemplarische Analyse seiner Familieninteraktionen zeigt, dass dort eine anregungsarme und unterrichtlich wenig anschlussfähige kommunikative Erfahrungswelt auf vergleichsweise gering ausgebaute diskursive Kompetenzen des Kindes v. a. auch im Bereich der Kontextualisierung stößt. Diese tragen ihrerseits dazu bei, dass die vollzogenen Gesprächskontexte wenig förderlich für den Erwerb diskursiver Kompetenzen sein dürften. Das Gattungsrepertoire seiner Familie erweist sich als äußerst schmal und umfasst vor allem so gut wie keine globalstrukturellen Diskursaktivitäten. Statt des ergebnisoffenen Argumentierens wie im Falle Alinas etwa finden sich lokale Praktiken (z. B. Frage-Antwort, Vorwurf-Rechtfertigung), die einseitig auf elterliche Entscheidungen und Deutungen gerichtet sind. Das Interaktionsmuster Dulden und Fallenlassen etabliert gerade keine globalen Anforderungen (etwa zum Argumentieren), sondern nutzt verschiedenste Verfahren des Hinaussteuerns aus potenziell argumentativen Kontexten (z. B. Ignorieren, Suspendieren, Insistieren). In der Folge ergeben sich keine strukturell komplexen Argumentationen, sondern rein lokal-sequenziell gebundene Aneinanderreihungen v. a. impliziter Begründungen sowie nicht genuin argumentativer Mittel (z. B. Widerspruch, Insistieren, Betteln). Stefan erlangt auf diese Weise weder produktiv noch rezeptiv Zugang zu Praktiken der Vertextung und expliziten Markierung diskursiver Strukturen.

Das elternseitige Disalignment (z. B. Stöhnen, no-knowlegde-reponses) und die zahlreichen - für Alltagsgespräche z. T. ungewöhnlich langen - zeitlichen Verzögerungen (Beispiel 2, z. B. Z. 31, 37, 40, 43, 46, 49) weisen dabei auf eine gewisse ,Schwerfälligkeit‘ des (potenziell) argumentativen Austausches hin. Dies legt den Schluss nahe, dass den Beteiligten zur argumentativen Bewältigung der Entscheidungsfindung keine routinierten Verfahren zur Verfügung stehen, auf die standardmäßig zurückgegriffen werden kann - und zwar auch den beteiligten Erwachsenen nicht. Wie auch für familiales Erklären mikroanalytisch rekonstruiert (Morek 2016b), scheinen sich darin ggf. eingeschränkte Diskurskompetenzen auch auf Erwachsenenseite bemerkbar zu machen, die es 
den Eltern erschweren, modellierend oder interaktiv unterstützend entsprechende Interaktionen tragen und Kindern implizite Hilfen für den Erwerb liefern zu können. ${ }^{30}$

Insgesamt ergibt sich so eine Konstellation, die man durchaus als tragisch bezeichnen könnte: Erstens ist Stefan aufgrund der eingeschränkten elterlichen Diskursstrukturierung und Responsivität auf eigene Initiativen angewiesen, wenn überhaupt Diskursaktivitäten zur Bearbeitung bestimmter kommunikativer Ziele in Gang kommen sollen. Anders als die ohnehin schon deutlich diskurskompetente Alina erhält er dabei keine interaktive Unterstützung. Unter Erwerbsgesichtspunkten bedeutet dies, dass er nicht unterstützt in seiner „Zone der nächstfolgenden Entwicklung“ (Vygotski 1978; s.a. Bruner 1987) agieren kann, sondern im Grunde weit darüber hinaus gehen müsste, wenn er ,aus eigener Kraft' Kontexte z. B. des Argumentierens schaffen wollte. Zweitens führen die Erfordernisse der sozialen Kontextualisierung im Falle Stefans dazu, dass ein etwaiges Argumentieren von einer Präferenz für nicht-argumentative Praktiken überformt wird (z. B. Bitte um Rat oder Erlaubnis, Betteln). Im Zuge der Orientierung am Üblichen des familialen kommunikativen Repertoires gibt es damit keine Chancen, sequenzielle Kontexte z. B. für echtes Argumentieren und den Ausbau entsprechender Diskursfähigkeiten mitzuschaffen.

Im Rahmen seiner Cliquen-Interaktionen treffen die eingeschränkten diskursiven Kompetenzen Stefans nun zudem auf einen - im Vergleich mit anderen Cliquen - unter Erwerbsgesichtspunkten ebenfalls diskursiv wenig fordernden und wenig anregungsreichen Kontext. Ganz anders als in den Cliquen aus sozial privilegierten Mitgliedern (z. B. Alinas Clique) wird dort - wie schon in seiner Familie - nur ein kleiner Teil der gemeinsamen Interaktionszeit auf global-strukturelle Diskursaktivitäten verwendet - und an diesen hat Stefan dazu kaum jemals aktiv verbal teil. Die exemplarische Analyse hat am Beispiel einer vermeintlichen Erklärsequenz gezeigt, dass bestimmte kommunikative Probleme - z. B. das kooperative Explizieren von Wissensbeständen zur Behebung von Wissensdefiziten typischerweise als solche gar nicht etabliert werden. Stattdessen werden mittels ironisierender und stilisierender Verfahren sogar sichtbar Distanzierungen von einem ,echten' Erklären vorgenommen, die den möglichen sequenziellen Weg in explanative Diskurseinheiten hinein verstellen. Somit ergibt sich auch kein Anwendungsraum für gattungsspezifische Vertextungen oder Markierungen,

$30 \mathrm{Zu}$ interindividuellen Differenzen in der Sprach- und Diskurskompetenz erwachsener Sprecherinnen und Sprecher ist in der Sprach- und Diskurserwerbsforschung bislang so gut wie nichts bekannt (vgl. aber Dąbrowska 2012). 
die übertragbar wären etwa auf unterrichtliche Kontexte. Zudem blockiert die in weiten Teilen spielerisch-aggressiv und kompetitiv angelegte Interaktion der „Party Boys” fordernd-unterstützende Gesprächsaktivitäten der Peers untereinander. Stefans Cliquen-Interaktion mag somit u. U. durchaus einen Erwerbskontext bieten für anspruchsvolle interaktive Aktivitäten wie den Umgang mit Ironie, Witz und Schlagfertigkeit (vgl. dazu auch Morek 2015). Diese können jedoch kaum für unterrichtliche Kontexte fruchtbar gemacht werden. Hinzukommt, dass gerade der Umgang mit gebrochenen oder ambigen Interaktionsmodalitäten besonders hohe Anforderungen an die Kontextualisierungsleistungen der Beteiligten stellt (Heller 2016). An diesen scheint ein Kind wie Stefan, dessen Diskurs- und v. a. Kontextualisierungskompetenz wenig ausgebaut ist, zu scheitern. So ist auch zu erklären, dass er kaum jemals mit verbalen Beiträgen an der Peer-Interaktion partizipiert.

\section{Fazit}

Ziel des vorliegenden Beitrags war es, alltägliche Interaktionserfahrungen unterschiedlicher Kinder in ihren Familien und Cliquen vergleichend in den Blick zu nehmen und dabei v. a. deren Potenzial für den kindlichen Erwerb unterrichtsrelevanter diskursiver Fähigkeiten zu beleuchten. Datenbasis waren Aufzeichnungen familialer Tischgespräche und informell-spontaner Cliquengespräche derselben Fünftklässler, die im Projekt DisKo erhoben wurden. Dabei förderte bereits die Erfassung der darin jeweils instanziierten sprachlich-kommunikativen Praktiken in Form von Gattungsrepertoires beträchtliche Variabilität der außerschulischen Kommunikationswelten von Präadoleszenten zutage. In Übereinstimmung mit bisherigen Befunden zu familialen Eltern-Kind-Gesprächen zeigt sich, dass Familien bei Tisch unterschiedlich breite Repertoires an sprachlichen und diskursiven Aktivitäten aktualisieren (Heller 2012). Dabei wird die Tendenz sichtbar, dass Gattungsrepertoires in sozial privilegierten Familien eher breit sind und v. a. stets die unterrichtlich besonders relevanten global strukturierten Gattungen des Erzählens, Argumentierens und Erklärens umfassen. Für sozial benachteiligte Familien dagegen lässt sich eine Tendenz zu vergleichsweise schmaleren Repertoires feststellen und v. a. das gänzliche Fehlen der vorgenannten drei Diskursgattungen in einigen Familien. Diese Beobachtungen sind kompatibel mit der von Quasthoff et al. (2015: 221) festgestellten vergleichsweise größeren Heterogenität der Praktiken unter sozial benachteiligten Familien. Insgesamt eröffnen sich Kindern so in ihren familialen Interaktionen unterschiedlich weite kommunikative Erfahrungsräume.

Der in der bisherigen Forschung kaum jemals vorgenommene direkte Vergleich verschiedener Cliquen-Interaktionen (vgl. aber Eder 1990) offenbart darüber 
hinaus, dass milieubedingte Disparitäten dort noch deutlicher zutage treten als im Familienvergleich. Wiederum sind es hier die Cliquen aus sozial privilegierten Mitgliedern, deren Repertoires sprachlich-diskursiver Praktiken mannigfaltiger sind und die wesentlich mehr Gesprächszeit v. a. für narrative Aktivitäten, aber auch für explanative und argumentative Aktivitäten aufwenden. Während dort also global strukturierte Praktiken mit Potenzial zu unterrichtlicher Anschlussfähigkeit fester Teil der Peer-Routinen sind, überwiegen in den Cliquengesprächen sozial benachteiligter Kinder lokal organisierte und stark kontextgebundene Aktivitäten. In den meisten dieser Cliquen finden sich keine erklärenden oder argumentativen Sequenzen und selbst erzählende nur selten. Vor diesem Hintergrund muss die Annahme, dass auch Peer-Interaktionen ein Erwerbskontext für bildungssprachlich relevante, diskursive Fähigkeiten wie Erklären und Argumentieren sein können (Zadunaisky, Ehrlich \& Blum-Kulka 2010) relativiert, mindestens aber differenziert werden: Offenbar gilt dies nur in eingeschränktem Maße für sozial benachteiligte Kinder. Dies bedeutet zugleich, dass diese Kinder wesentlich stärker auf die Familie als alleinigen außerschulischen Diskurserwerbskontext angewiesen sind. Insofern liefern diese Befunde ein zusätzliches Argument dafür, auf die Stärkung elterlichen Interaktions- und Unterstützungsverhaltens zu setzen, wie es im EfA-Projekt (Kluger \& Quasthoff in diesem Band) verfolgt wird.

Der gesprächsanalytische Zugriff auf interaktive und sprachliche Strukturen diskursiver Sequenzen ausgewählter Familien- und Peer-Gespräche zeigt darüber hinaus, dass und wie solche an der Oberfläche von Interaktionen aufscheinenden Varianzen von den Beteiligten hervorgebracht werden. Im gesprächsanalytischen Mikroskop ließ sich dabei vorführen, wie der interaktive Vollzug und die Nutzung gattungsspezifischer Vertextungs- und Sprachstrukturen überformt wird von und in Zusammenhang steht mit familien- bzw. cliquenspezifischen Kommunikationskulturen. Auch wurde auf diese Weise ersichtlich, wie die internen diskursiven Ressourcen der Beteiligten - der betreffenden Kinder, aber letztlich auch der jeweiligen Erwachsenen - ihrerseits wieder an der Hervorbringung bestimmter Gesprächs- und damit Diskurserwerbskontexte beteiligt sind. Dies spricht für eine grundsätzliche Dynamisierung des Verständnisses von ,Erwerbskontext“ insbesondere mit Blick auf die hier fokussierten späteren Phasen des Diskurserwerbs (Präadoleszenz und darüber hinaus). Ein Erwerbskontext (vgl. Quasthoff et al. 2019) ist somit weder einfach ,gegeben' noch wird er allein von den ,kompetenteren Anderen' konfiguriert, sondern Kinder und Jugendliche prägen sehr wesentlich mit, welche Erwerbskontexte sich ihnen bieten. Um das Zusammenspiel verschiedener sozialer Kontexte und interner und externer Ressourcen beim Erwerb komplexer diskursiver Kompetenzen en detail nachzuzeichnen, sind qualitativ-explorativ angelegte Studien, die die Beobachtung von Kindern in verschiedenen Kontexten ermöglichen, ebenso notwendig wie vielversprechend. 


\section{Literatur}

Arendt, Birte (2015): Kindergartenkinder argumentieren - Peer-Gespräche als Erwerbkontext. Mitteilungen des Deutschen Germanistenverbandes 62 (1), 21-33.

Arnon, Inbal, Marisa Casillas, Chigusa Kurumada \& Bruno Estigarribia (Hrsg.) (2014): Language in interaction. Studies in honor of Eve V. Clark. Amsterdam: Benjamins.

Auer, Peter (1986): Kontextualisierung. Studium Linguistik 19, 22-47.

Auer, Peter (2003): ,Türkenslang‘: Ein jugendsprachlicher Ethnolekt des Deutschen und seine Transformationen. In Annelies Häcki Buhofer \& Lorenz Hofer (Hrsg.), Spracherwerb und Lebensalter, 255-264. Tübingen: Francke.

Behrens, Heike (Hrsg.) (2014): Reflections: 40 Years of JCL. Special Issue. Journal of Child Language, 41, Supplement 1.

Blum-Kulka, Shoshana (1997): Dinner talk. Patterns of sociability and socialization in family discourse. Mahwah, NJ: Erlbaum.

Blum-Kulka, Shoshana \& Catherine Snow (2004): Introduction: The Potential of Peer Talk. Discourse Studies 6 (3), 291-306.

Bonsen, Martin, Wilfried Bos, Carola Gröhlich \& Heike Wendt (2008): Bildungsrelevante Ressourcen im Elternhaus: Indikatoren der sozialen Komposition der Schülerschaften an Dortmunder Schulen. In Stadt Dortmund - Der Oberbürgermeister (Hrsg.), Erster kommunaler Bildungsbericht für die Schulstadt Dortmund. Schulentwicklung in Dortmund, 125-149. Münster [u. a.]: Waxmann.

Bruner, Jerome (1983): Child's talk. Learning to use language. New York: Norton.

Button, Graham (1987): Moving out of closings. In Graham Button \& John Lee (Hrsg.), Talk and social organisation, 101-151. Clevedon: Multilingual Matters.

Cekaite, Asta, Shoshana Blum-Kulka, Vibeke Grøver \& Eva Teubal (Hrsg.) (2014): Children's peer talk. Learning from each other. Cambridge: Cambridge University Press.

Clark, Eve (2017): Language in children. Milton Park [u. a.]: Routledge.

Clayman, Steven (2002): Sequence and solidarity. In Shane Thye \& Edward Lawler (Hrsg.), Group cohesion, trust and solidarity, 229-253. Amsterdam, Oxford: JAl.

Dąbrowska, Ewa (2012): Different speakers, different grammars. Linguistic Approaches to Bilingalism 2 (3), 219-253.

Deppermann, Arnulf \& Axel Schmidt (2001): ,Dissen‘: Eine interaktive Praktik zur Verhandlung von Charakter und Status in Peer-Groups männlicher Jugendlicher. Osnabrücker Beiträge zur Sprachtheorie 62, 79-98.

Duranti, Alessandro, Elinor Ochs \& Bambi Schieffelin (Hrsg.) (2012): The Handbook of Language Socialization. Malden, MA: Wiley.

Eder, Donna (1990): Serious and Playful Disputes: Variation in Conflict Talk among Female Adolescents. In Allen Day Grimshaw (Hrsg.), Conflict talk. Sociolinguistic investigations of arguments in conversations, 67-84. Cambridge, New York: Cambridge University Press.

Feilke, Helmuth (2012): Bildungssprachliche Kompetenzen - fördern und entwickeln.

Basisartikel. Praxis Deutsch 233, 4-13.

Garfinkel, Harold (1967): Studies in ethnomethodology. Englewood Cliffs, N.J: Prentice-Hall.

Goodwin, Marjorie (2006): The hidden life of girls. Games of stance, status, and exclusion. Malden, MA: Blackwell. 
Goodwin, Marjorie \& Charles Goodwin (1987): Children's Arguing. In Susan Urmston Philips, Susan Steele \& Christine Tanz (Hrsg.), Language, gender and sex in comparative perspective, 200-248. Cambridge, New York: Cambridge University Press.

Grundler, Elke \& Rüdiger Vogt (2013): Mündliche Argumentationskompetenz im Primarbereich sowie in den Sekundarstufen I und II. In Steffen Gailberger \& Frauke Wietzke (Hrsg.), Handbuch Kompetenzorientierter Deutschunterricht, 456-490, Weinheim, Basel: Beltz.

Gumperz, John (1982): Discourse strategies. Cambridge [u. a.]: Cambridge University Press.

Günthner, Susanne (2009): Intercultural communication and the relevance of cultural specific repertoires of communicative genres. In Helga Kotthoff \& Helen Spencer-Oatey (Hrsg.), Handbook of intercultural communication, 127-152. Berlin: De Gruyter.

Günthner, Susanne \& Gabriela Christmann (1996): Entrüstungs- und Mokieraktivitäten Kommunikative Gattungen im Kontextvergleich. Folia Linguistica 30 (3-4), 327-357.

Hart, Betty \& Todd Risley (1995): Meaningful Differences in the Everyday Experience of Young American Children. Baltimore: Brookes.

Hausendorf, Heiko \& Uta Quasthoff (1996): Sprachentwicklung und Interaktion. Eine linguistische Studie zum Erwerb von Diskursfähigkeiten. Opladen: Westdeutscher Verlag.

Heath, Shirley Brice (1983): Ways with words. Language, life, and work in communities and classrooms. Cambridge, New York: Cambridge University Press.

Heath, Shirley Brice (2006): Building the micros toward seeing the macro. Text \& Talk 26 (4-5), 627-634.

Heller, Vivien (2012): Kommunikative Erfahrungen von Kindern in Familie und Unterricht. Passungen und Divergenzen. Tübingen: Stauffenburg.

Heller, Vivien (2016): „das_s VOLL verARsche hier“: Aligment und Disalignment mit jugendsprachlichen Praktiken in der Unterrichtsinteraktion. In Carmen Spiegel \& Daniel Gysin (Hrsg.), Jugendsprache in Schule, Medien und Altag, 91-108. Frankfurt am Main: Lang.

Heller, Vivien (2018): Embodying epistemic responsibility. The interplay of gaze and stancetaking in children's collaborative reasoning. Research on Children and Social Interaction 2 (2), 262-285.

Heller, Vivien \& Antje Krah (2015): Wie Eltern und Kinder argumentieren. Interaktionsmuster und ihr erwerbssupportives Potenzial im längsschnittlichen Vergleich. Mitteilungen des Deutschen Germanistenverbandes 62 (1), 5-20.

Heller, Vivien, Uta Quasthoff, Anna Vogler \& Susanne Prediger (2017): Bildungssprachliche Praktiken aus professioneller Sicht: Wie deuten Lehrkräfte Erklärungen und Begründungen von Kindern? In Bernt Ahrenholz, Britta Hövelbrinks \& Claudia Schmellentin (Hrsg.), Fachunterricht und Sprache in schulischen Lehr-/Lernprozessen, 139-160. Tübingen: Narr.

Hoffmann, Ludger (2013): Deutsche Grammatik. Grundlagen für Lehrerausbildung, Schule, Deutsch als Zweitsprache und Deutsch als Fremdsprache. Berlin: Schmidt.

Isler, Dieter (2014): Vorschulischer Erwerb von Literalität in Familien: Erkundungen im Mikrokosmos sprachlicher Praktiken und Fähigkeiten von 5- und 6-jährigen Kindern. Dissertation, Universität Genf. Online verfügbar unter http://archive-ouverte.unige.ch/ unige:40025. (Letzter Zugriff am 26. 06.2020)

Jefferson, Gail (1988): On the Sequential Organization of Troubles-Talk in Ordinary Conversation. Social Problems 35 (4), 418-444.

Keppler, Angela (1994): Tischgespräche. Über Formen kommunikativer Vergemeinschaftung am Beispiel der Konversation in Familien. Frankfurt am Main: Suhrkamp. 
Keppler, Angela \& Thomas Luckmann (1991): Teaching: Conversational transmission of knowledge. In Ivana Markovà \& Klaus Foppa (Hrsg.), Asymmetries in dialogue, 143-166. Savage: Barnes \& Noble Books.

Kern, Friederike (2003): Bedeutung und Interaktion: Spielerklärungen bei Kindem. In Stefanie Haberzettl \& Heide Wegener (Hrsg.), Spracherwerb und Konzeptualisierung, 257-270, Frankfurt am Main, New York: Lang.

Kern, Friederike (2011): Der Erwerb kommunikativer Praktiken und Formen - Am Beispiel des Erzählens und Erklärens. In Stephan Habscheid (Hrsg.), Textsorten, Handlungsmuster, Oberflächen. Linguistische Typologie der Kommunikation, 231-253. Berlin: De Gruyter.

Kern, Friederike \& Uta Quasthoff (2005): Fantasy stories and conversational narratives of personal experience: Genre-specific, interactional and developmental aspects. In Uta Quasthoff \& Tabea Becker (Hrsg.), Narrative interaction, 15-56. Amsterdam, Philadelphia: Benjamins.

Kern, Friederike \& Uta Quasthoff (2007): Familiale Interaktionsmuster und kindliche Diskursfähigkeit: Mögliche Auswirkungen interaktiver Stile auf diskursive Praktiken und Kompetenzen bei Schulkindern. In Heiko Hausendorf (Hrsg.), Gespräch als Prozess. Linguistische Aspekte der Zeitlichkeit verbaler Interaktion, 277-306. Tübingen: Narr.

Klein, Josef (2009): ERKLÄREN-WAS, ERKLÄREN-WIE, ERKLÄREN-WARUM. Typologie und Komplexität zentraler Akte der Welterschließung. In Rüdiger Vogt (Hrsg.), Erklären. Gesprächsanalytische und fachdidaktische Perspektiven, 25-36. Tübingen: Stauffenburg.

Klein, Wolfgang (1980): Argumentation und Argument. Zeitschrift für Literaturwissenschaft und Linguistik 38/39, 9-57.

Kleinschmidt-Schinke, Katrin (2018): Die an die Schüler/-innen gerichtete Sprache (SgS). Studien zur Veränderung der Lehrer/-innensprache von der Grundschule bis zur Oberstufe. Berlin: De Gruyter.

Kotthoff, Helga (2015): Konsensuelles Argumentieren in schulischen Sprechstunden. In Stefan Hauser \& Vera Mundwiler (Hrsg.), Sprachliche Interaktion in schulischen Elterngesprächen, 72-98. Bern: hep.

Kurumada, Chigusa \& Inbal Arnon (2014): Introduction: Language acquisition in interaction. In Arnon Inbal, Marisa Casillas, Chigusa Kurumada \& Bruno Estigarribia (Hrsg.), Language in Interaction, 1-10. Amsterdam: Benjamins.

Labov, William \& Joshua Waletzky (1967): Narrative Analysis. In June Helm (Hrsg.), Essays on the verbal and visual arts. Proceedings of the 1966 annual spring meeting of the American Ethnological Society, 12-44. Seattle: University of Washington Press.

Lareau, Annette (2003): Unequal childhoods. Class, race, and family life. Berkeley: University of California Press.

Luckmann, Thomas (1989): Kultur und Kommunikation. In Max Haller, Wolfgang Zapf \& HansJoachim Hoffmann-Nowotny (Hrsg.), Kultur und Gesellschaft, 33-45. Frankfurt: Campus.

Morek, Miriam (2012): Kinder erklären. Interaktionen in Familie und Unterricht im Vergleich. Tübingen: Stauffenburg.

Morek, Miriam (2014): Constructing social and communicative worlds - The role of peerinteractions in preadolescents' discursive development. Learning, Culture and Social Interaction 3 (2), 121-133.

Morek, Miriam (2015): Dissensbearbeitung unter Gleichaltrigen - (k)ein Kontext für den Erwerb argumentativer Gesprächsfähigkeiten? Mitteilungen des Deutschen Germanistenverbandes 62 (1), 34-46. 
Morek, Miriam (2016a): Formen mündlicher Darstellung in situ: Zur Komplexität von

Diskursanforderungen in Unterrichtsgesprächen. In Ulrike Behrens \& Olaf Gätje (Hrsg.), Mündliches und schriftliches Handeln im Deutschunterricht. Wie Themen entfaltet werden, 95-131. Frankfurt am Main: Lang.

Morek, Miriam (2016b): ,watt soll ich dazu SAgen“ - (Dis)Alignment bei der interaktiven Manifestation epistemischer Asymmetrien. In Alexandra Groß \& Inga Harren (Hrsg.), Wissen in institutioneller Interaktion, 145-175. Frankfurt am Main: Lang.

Morek, Miriam (2020): Learning to modalize is learning to reason - On the role of epistemic modalizations in parent-child-talk and in written argumentation of secondary school students. Research on Children and Social Interaction 4 (1), 115-141.

Morek, Miriam, Vivien Heller \& Uta Quasthoff (2017): Erklären und Argumentieren Modellierungen und empirische Befunde im Rahmen der linguistischen Erwerbs- und Unterrichtsforschung. In Iris Meißner \& Eva Wyss (Hrsg.), Begründen - Erklären Argumentieren. Konzepte und Modellierungen in der Angewandten Linguistik, 11-46. Tübingen: Stauffenburg.

Morek, Miriam \& Uta Quasthoff (2018): Sprachliche und diskursive Praktiken unter Kindern. In Eva Neuland \& Peter Schlobinski (Hrsg.), Handbuch Sprache in sozialen Gruppen, 255-275. Berlin, Boston: De Gruyter.

Nardy, Aurélie, Jean-Pierre Chevrot \& Stéphanie Barbu (2013): The acquisition of sociolinguistic variation. Looking back and thinking ahead. Linguistics 51 (2), 255-284.

Nelson, Katherine (2014): A matter of meaning: reflections on forty years of JCL. Journal of child language 41, Supplement 1, 93-104.

Nippold, Marilyn (2006): Later language development. School-age children, adolescents, and young adults. Austin: PRO-ED.

Ochs, Elinor \& Bambi Schieffelin (2012): The Theory of Language Socialization. In Alessandro Duranti, Elinor Ochs \& Bambi Schieffelin (Hrsg.), The Handbook of Language Socialization, 1-19. Malden: Wiley.

Ohlhus, Sören \& Juliane Stude (2009): Erzählen im Unterricht der Grundschule. In: Michael Becker-Mrotzek (Hrsg.), Mündliche Kommunikation und Gesprächsdidaktik, 471-486. Baltmannsweiler: Schneider.

Paugh, Amy (2016): Language Socialization. In Nancy Bonvillain (Hrsg.), The Routledge Handbook of Linguistic Anthropology, 125-139. New York: Routledge.

Pohl, Thorsten (2014): Schriftliches Argumentieren. In Helmuth Feilke \& Thorsten Pohl (Hrsg.), Schriftlicher Sprachgebrauch. Texte verfassen, 287-315. Baltmannsweiler: Schneider Hohengehren.

Quasthoff, Uta (1985): Argumentationsbarrieren. Die Manifestation von Gruppenspezifik und die Behinderung von Verständigung durch topisches Argumentieren. In Josef Kopperschmidt \& Helmut Schanze (Hrsg.), Argumente - Argumentation. Interdisziplinäre Problemzugänge, 170-207. München: Fink.

Quasthoff, Uta (2011): Diskurs- und Textfähigkeiten: Kulturelle Ressourcen ihres Erwerbs. In Ludger Hoffmann, Kerstin Leimbrink \& Uta Quasthoff (Hrsg.), Die Matrix der menschlichen Entwicklung, 210-251. Berlin: De Gruyter.

Quasthoff, Uta (2012): Aktual- und mikrogenetische Zugänge zur Ontogenese: Inspirationen der Konversationsanalyse zur Verbindung von sprachlichen Praktiken und dem Erwerb sprachlicher Kompetenzen. In Ruth Ayaß \& Christian Meyer (Hrsg.), Sozialität in Slow Motion. Theoretische um empirische Perspektiven. Festschrift für Jörg Bergmann, 217-244. Wiesbaden: Springer. 
Quasthoff, Uta \& Dietrich Hartmann (1982): Bedeutungserklärungen als empirischer Zugang zu Wortbedeutungen. Zur Entscheidbarkeit zwischen holistischen und komponentiellen Bedeutungskonzeptionen. Deutsche Sprache 10, 97-118.

Quasthoff, Uta, Vivien Heller \& Miriam Morek (2017): On the sequential organization and genre-orientation of discourse units in interaction: An analytic framework. Discourse Studies 19 (1), 84-110.

Quasthoff, Uta, Friederike Kern, Sören Ohlhus \& Juliane Stude (2019): Diskurse und Texte von Kindern: Praktiken - Fähigkeiten - Ressourcen: Erwerb. Tübingen: Stauffenburg.

Quasthoff, Uta \& Antje Krah (2012): Familiale Kommunikation als Spracherwerbsressource: das Beispiel argumentativer Kompetenzen. In Eva Neuland (Hrsg.), Sprache der Generationen. Thema Deutsch Band 12, 115-132. Mannheim: Bibliographisches Institut.

Quasthoff, Uta \& Miriam Morek (2015): Abschlussbericht zum DFG-Projekt „Diskursive Praktiken von Kindern in außerschulischen und schulischen Kontexten". Manuskript. TU Dortmund. Online verfügbar unter www.disko.tu-dortmund.de/disko/Medienpool/ Abschlussbericht-DisKo.pdf. (Letzter Aufruf am 26.06.2020)

Quasthoff, Uta, Elke Wild, Madeleine Domenech, Jelena Hollmann, Christian Kluger \& Antje Krah (2015): Mündliches und schriftliches Argumentieren in der Sekundarstufe I: Können Eltern ihre Kinder beim Erwerb dieser Schlüsselkompetenz unterstützen? In Angelika Redder, Johannes Naumann \& Rosemarie Tracy (Hrsg.), Forschungsinitiative Sprachdiagnostik und Sprachförderung - Ergebnisse, 68-88. Münster: Waxmann.

Rehbein, Jochen (1984): Beschreiben, Berichten und Erzählen. In Konrad Ehlich (Hrsg.), Erzählen in der Schule, 67-124. Tübingen: Narr.

Schieffelin, Bambi \& Elinor Ochs (1986): Language socialization. Annual Review of Anthropology 15, 163-191.

Schleppegrell, Mary (2004): The language of schooling. A functional linguistics perspective. Mahwah: Erlbaum.

Schlobinski, Peter, Gaby Kohl \& Irmgard Ludewigt (1993): Jugendsprache. Fiktion und Wirklichkeit. Opladen: Westdeutscher Verlag.

Schmidt, Axel (2004): Doing peer-group. Die interaktive Konstitution jugendlicher Gruppenpraxis. Frankfurt am Main [u. a.]: Lang

Selting, Margret, Peter Auer, Dagmar Barth-Weingarten, Jörg Bergmann, Pia Bergmann, Karin Birkner et al. (2009): Gesprächsanalytisches Transkriptionssystem 2 (GAT 2). Gesprächsforschung - Online-Zeitschrift zur verbalen Interaktion 10, 353-402. Online verfügbar unter http://www.gespraechsforschung-ozs.de/heft2009/px-gat2.pdf. (Letzter Aufruf am 17.09.2020).

Slobin, Dan, Katya Tripp \& Gail Silverman (Hrsg.) (1996): Social interaction, social context, and language. Essays in honor of Susan Ervin-Tripp. New York: Psychology Press.

Snow, Catherine (1977): Mother's Speech Research: from Input to Interaction. In Catherine Snow \& Charles Ferguson (Hrsg.), Talking to Children. Language Input and Acquisition, 31-49. Cambrigde: Cambridge University Press.

Snow, Catherine (2014): Input to interaction to instruction: three key shifts in the history of child language research. Journal of child language 41 Supplement 1, 117-123.

Spiegel, Carmen (2003): „zum beispiel es gibt ja leute . . .“- Das Beispiel in der Argumentation Jugendlicher. In Arnulf Deppermann \& Martin Hartung (Hrsg.), Argumentieren in Gesprächen. Gesprächsanalytische Studien, 111-129. Tübingen: Stauffenburg. 
Stoll, Sabine (2016): Studying Language Acquisition in Different Linguistic and Cultural Settings. In Nancy Bonvillain (Hrsg.), The Routledge Handbook of Linguistic Anthropology, 140-158. New York: Routledge.

Stude, Juliane (2003): Mündliche und schriftliche Spielerklärungen von Grundschulkindern erste Analysen des OLDER-Korpus. (OLDER-Projektpapier Nr. 3). Manuskript, TU Dortmund.

Stude, Juliane (2013): Kinder sprechen über Sprache. Eine Untersuchung zu interaktiven Ressourcen des frühen Erwerbs metasprachlicher Kompetenz. Stuttgart: Fillibach.

Uccelli, Paola, Christopher Barr, Christina Dobbs, Emily Phillips Galloway, Alejandra Meneses \& Emilio Sánchez (2014): Core academic language skills: An expanded operational construct and a novel instrument to chart school-relevant language proficiency in preadolescent and adolescent learners. Applied Psycholinguistics 36, 1077-1109.

Vygotsky, Lev (1978): Mind in society. The development of higher psychological processes. Cambridge: Harvard University Press.

Walther, Diana (2014): Scherzkommunikation unter Jugendlichen. Lästern, Frotzeln und Blödeln in gemischtgeschlechtlichen Kleingruppen. Frankfurt am Main: Lang.

Weinrich, Harald, Maria Thurmair, Eva Breindl \& Eva-Maria Willkop (2007): Textgrammatik der deutschen Sprache. Hildesheim, New York: Olms.

Zadunaisky Ehrlich, Sara \& Shoshana Blum-Kulka (2010): Peer talk as a ,double opportunity space': The case of argumentative discourse. Discourse \& Society 21 (2), 211-233.

Zifonun, Gisela, Ludger Hoffmann \& Bruno Strecker (1997): Grammatik der deutschen Sprache. Berlin [u. a.]: De Gruyter. 